doi:10.29285/actapinteriana.2019.5.91

\title{
Il gioco come simbolo del mondo creato \\ Verso una teologia ludica
}

\author{
Bagyinszki Péter Ágoston OFM \\ Sapientia Szerzetesi Hittudományi Főiskola, 1052 Budapest, Piarista köz 1. \\ bagyinszki.agoston@sapientia.hu
}

Bagyinszki P. Á. (2018): Il gioco come simbolo del mondo creato. Verso una teologia ludica. A játék mint a teremtett világ szimbóluma. Egy ludikus teológia felé. Acta Pintériana, 5: 91-125.

Összegzés: A tanulmány első része a játék jelentésrétegeit különíti el, kitér a játékok Caillois-féle klasszikus osztályozási rendszerére, szembenéz az alapvető definíciós problémával. A játék értelmezhető mint a világ elhatárolt eseménye, mint az ember belső magatartása, és mint ontológiai karakterjegy. A tanulmány második része J. Huizinga és H. Rahner immár klasszikus dialógusát tárgyalja, amelyben a homo ludens téma antropológiai és teológiai szempontjai kerülnek kapcsolatba egymással. A harmadik fejezet a ludikus szemléletmód posztmodern beágyazottságát és a theologia ludica mai lehetőségeit veszi szemügyre. Végül a negyedik fejezet rámutat a Deus ludens - homo ludens diptichon hagyományban gyökerező intuitív erejére, valamint kritikával illeti a komor komolyság és a cinizmus hamis dilemmáját.

Il gioco come argomento filosofico e teologico - nonostante si basi sull'analisi di un sistema di simboli enormemente ricco svolgente un ruolo importante nella storia delle civiltà - è poco noto. Nella filosofia i commentatori del frammento 52 di Eraclito scoprono di nuovo la filosofia del gioco mentre nella storia della teologia Proverbi 8, 30-31 con suoi commenti raggruppa gli studi sulla simbolica del gioco e tutti e due i rami della tradizione vedono coinvolti i nomi più famosi della storia delle scienze. Circa ottanta anni fa tra Johann Huizinga e Hugo Rahner si è svolto un dialogo fondamentale di carattere filosofico-teologico riguardo all'homo ludens, il quale alla luce della letteratura allora esistente sull'argomento può essere valutato nel senso teologico tramite la recensione dei libri dei due autori, legati a quest'argomento.

\section{Cosa è il gioco? Se nessuno me lo domanda lo so, ma quando cerco di spiegarlo a qualcuno non lo so più...}

Il concetto chiave della nostra riflessione teologica sul gioco può essere basato sulla simbolica del gioco e sugli approcci connessi al gioco ma ne può far parte anche. Incominceremo con alcuni brevi cenni sulla simbolica del gioco, per offrire, poi, un riassunto degli approcci connessi al gioco e, infine, comporre le conclusioni filosofiche della lettaratura sul gioco.

\subsection{La famiglia di parole e la simbolica del gioco}

Il Grande Dizionario della Lingua Ungherese distingue ventidue gruppi semantici della parola „gioco" (A Magyar Nyelv Értelmezö Szótára [Grande Dizionario della Lingua Ungherese] 1986). Il 
„gioco" può significare un oggetto con cui si gioca, un'attività compiuta per diletto o per capriccio, un modo di agire a volte con rischio o con astuzia per raggiungere uno scopo pratico, il moto o la possibilità di moto (anche nel caso di un oggetto), ecc.

Ecco alcuni esempi della estesa famiglia della parola „gioco" nella lingua ungherese: debito di gioco, automobile da gioco, bambola (da gioco), negozio di giocattoli, concetto di gioco, pellicola a soggetto (in ungherese: ,játékfilm”), carta da gioco, casinò da gioco, maestro di gioco, modo di giocare, giocatore, regola del gioco, divieto di gioco, arbitro (di gioco), gioco di parole... I verbi „giocare" e "giocherellare" allargano ancora il campo dei significati legati al gioco: effettuare un'attività a scopo di divertirsi; recitare in scena; fare movimento a dondolo; ecc... Anche al verbo „giocare” si agganciano altre parole, sempre della famiglia della parola „gioco”; p.es.: partita (in ungherese: ,játszma” deriva dal verbo ,játszani” - giocare), compagno di gioco, terreno di gioco. Tuttavia non basta esaminare il contesto linguistico del gioco solo nell' ambito della lingua ungherese ma gli va trovata una visione globale.

Nel Homo ludens Johann Huizinga dedica un intero capitolo all'internazionalità linguistica del gioco (HuIZINGA 1938; 1946). Oltre alle lingue europee passa in rassegna il latino, il greco, il sanscrito, il cinese, il giapponese, l'arabo, l'aramaico, l'ebraico, la lingua degli indiani „blackfoot”, e con l'aiuto di linguisti eccellenti analizza il lessico applicato per descrivere il fenomeno del gioco. Egli arriva alla conclusione che ogni popolo gioca e stranamente gioca in modo analogo ma tra tutte le lingue del mondo nessuna è in grado di esprimere in un unica parola il concetto del gioco così concreto e nello stesso tempo così generale come le lingue europee moderne (HUIZINGA 1946, p. 50). A questo possiamo aggiungere che la caratteristica generale delle lingue è che la parola „gioco" oltre a significare gioco di bambino sfiora anche i campi semantici della competizione, del ballo, della risata, dell'erotismo, della buffoneria, dell'illusione (in-lusio, in altre parole: entrare nel gioco).

Sotto l'aspetto linguistico multiforme del gioco si percepisce una simbolica del gioco avente profonde radici nella simbologia universale dell'umanità, rintracciabile sia nelle mitologie che nella letteratura. Huizinga accenna al gioco che si manifesta come modo di esistere degli dei mitologici e parla della loro rappresentazione come dei giocosi (ADRIANI 1967) mentre il filosofo e teorico del gioco Eugen Fink nei suoi libri Oasi della gioia (1957; 1969a) e Il gioco come simbolo del mondo $(1960 ; 1969 b)$ tratta le prospettive filosofiche del simbolismo legato al gioco. ${ }^{1}$

\subsection{Aspetti ideologici del gioco}

György Székely nella sua tesi di dottorato (1987), analizzando la storia sociale dell'arte teatrale, come principio base osserva che al contrario del genere drammatico letterario (i cui periodi importanti, com'è noto, nella letteratura universale sono brevissimi), le manifestazioni dell'attività teatrale dell'umanità sono presenti in tutte le culture ed hanno una continuità. Dal punto di vista del nostro tema questo significa che una certa autoriflessione giocosa è propria di ogni etnia dell'umanità - fin dai tempi preistorici - e avendo la sua continuità va considerata d'intonazione antropologica e socioantropologica (TURNER 1982; 1986). Le manifestazioni storiche della simbolica del gioco sono parte essenziale della simbologia collettiva (cfr. ELIADE 1987, pp. 363-367) e possono dare spazio ad o alcune interpretazioni d'importanza ideologica sul tema del motivo del gioco.

I mistici orientali e i filosofi greci per esprimere le loro esperienze intuitive sulla realtà fin dall'inizio usavano volentieri la metafora del gioco e della danza. Ne è un tipico esempio il sanscrito Lalitavistara (Trattato dettagliato sul gioco), la fonte piú importante sulla vita di Budda la quale rappresenta la vita terrestre del Sublime come il gioco di un essere ultraterreno (cfr. BELLINGER 1990,

\footnotetext{
${ }^{1}$ Nella seconda parte dell'articolo, in connessione con il cristianesimo parleremo dell'apertura del gioco verso la trascendenza (BERGER 1969), perciò qui proponiamo di saltare questa problematica.
} 
p. 75). ${ }^{2}$ Secondo la filosofia arcaica di Eraclito il tempo è un bambino che gioca a dama: la dominanza è del re bambino (Frammento 52); altrove paragona le opinioni umane ai giochi infantili. Il discorso sui problemi dell'esistenza secondo Parmenide è un ,gioco difficile”. Nel capitolo VII delle Leggi (303 e) Platone cerca la soluzione al problema seguente: in quale modo e „con quale strategia guidare in modo migliore la barca della nostra vita nel mare della vita?". L'uomo secondo Platone deve capire di non essere altro che un gioco nelle mani di Dio e deve accettare questa situazione dedicando la sua vita ai „giochi più belli”. Oggi invece ,si pensa che le cose serie siano in funzione dei divertimenti” ma in realtà è tutto il contrario. „Qual è allora la strada giusta? Dobbiamo vivere la nostra vita in mezzo ai giochi, giocando: facendo sacrifici, cantando, ballando, per poter ottenere la grazia degli dei, tenerci lontani i nemici e vincerli in lotta." Aristotele nel gioco ritiene importante la misura e scopre la virtù dell'eutrapelia. ${ }^{3}$ La ricca tradizione greca del gioco sopravvive nella letteratura mistica neoplatonica e cristiana primitiva esercitando un influsso notevole anche sulla mentalità cristiana medioevale.

Potrebbe sembrare che il motivo ludico sia un argomento periferico della filosofia antica ma in ogni caso è da notare che i padri della chiesa sentivano il bisogno di integrare l'idea del gioco delle filosofie pagane „battezzata” nella teologia cristiana. ${ }^{4}$ San Tommaso d'Aquino, eccellente sistematore degli studi teologici, nella Summa Theologiae (II-II, q. 168, aa. 2-4) risponde in tre articoli alle domande poste da Aristotele sul rapporto tra gioco, virtù, peccato e misura (THOMAS AQUINAS 1984); ma la teologia ludica godeva sempre una vera popolarità nelle celle dei mistici: il maestro Eckhart p.es. esalta Dio che „ride e gioca”.

Tra la fine del Medioevo e l'inizio dell'epoca moderna è proprio la mentalità del Rinascimento a scoprire con forza elementare il primitivo modello di vita del theatrum mundi e la dimensione profana giocosamente scherzosa del mondo. Secondo Huizinga, se una volta un'élite riservata e consapevole cercò di concepire la vita come un gioco al livello di perfezione artistica, non poteva essere altro che quella del Rinascimento. Tutta la pompa del Rinascimento è una sfilata allegra e solenne in maschere, nei costumi di un passato fantastico e ideale (cfr. HUIZINGA 1946, p. 224). Questo slancio giocoso che ha fatto nascere tra l'altro L'elogio della pazzia di Erasmo (1509) sembra incupire nei processi di desacralizzazione dell' Illuminismo e nella mentalità scientifica che dura tuttora portando con sé l'alienazione della simbologia del gioco dall' apertura verso la trascendenza.

Infine - parlando dei nostri tempi - Eberhard Straub (cfr. 1993, pp. 22-29) scrive sulla mentalità del gioco sfiorante il cinismo che nell'epoca postmoderna non soltanto il cristianesimo deve affrontare il disinteresse... È l'obiettivo della filosofia contemporanea di far valere le grandi tesi della storia sulla libertà e dignità dell'essere umano in connessione con la verità e non importa se questa verità è cristiana o storico-scientifica o la verità delle leggende che sembrano efficaci. Non sono altri che interessanti abbozzi poetici che concorrono fra di loro ma non si impediscono e non si disturbano. Convivono, uno accanto all'altro, indifferenti, nell'anarchia dei giochi delle parole, e ognuno è contento perché ogni gioco è coerente avendo il suo senso esclusivo nell'ambito delle sue regole. Questa pluralità dei giochi vuole dimostrare che l'uomo è libero solo dove gioca e dove festeggia... Questa mentalità può arrivare al punto di svelare la scorrettezza delle regole che non sono state costruite ,per il gioco”. Sia dal pensiero straubiano sia dalle opere elencate nella bibliografia possiamo

\footnotetext{
${ }^{2}$ La tradizione del gioco nella cultura semitica, insieme agli elementi di gioco presenti nella Bibbia saranno l'oggetto dell'analisi nel capitolo terzo, perciò qui si propone di esaminare le radici elleniche della cultura europea.

${ }^{3}$ In decem libros ethicorum Aristotelis ad Nicamachum expositio, I, X, lect. 9 e I. IV, lect. 16 (ARISTOTELIS 1949, 540 e 237). Eth. Nic. 10, 6, 1176 b; 4, 14, 1128 a; 2, 7, 1108 a.

${ }^{4}$ A proposito della tesi di Hugo Rahner sulla teologia patristica del gioco (RAHNER 1952; 1969) dovremo esaminare più in fondo i valori filosofici e teologici del gioco, qui peró si propone di continuare con l'ideologia del gioco.
} 
verificare l'importanza vitale ed esistenziale del paradigma dell' „essere per il gioco” nei nostri tempi. Il paradigma esistenziale dell' „essere per il gioco" non porta necessariamente a una concezione cinica aliena al cristianesimo ma riconducendolo alle radici sacro-ideologiche ci dà una prospettiva autentica-cristiana.

\subsection{Classificazione dei giochi e problemi della definizione}

Per rispondere alla domanda che cos'è il gioco potremmo usare il famoso aforisma agostiniano:

„Se nessuno me lo domanda lo so, ma quando cerco di spiegarlo a qualcuno non lo so più." (Conf. IX, 14)

Similmente al tempo, nel dare una definizione al gioco, il problema è: come coglierlo a livello di concetti, nella sua evidenza di realtà inevitabile? Anche il teorico del gioco Eugen Fink sottolinea le difficoltà della riflessione che si presentano nel caso del gioco:

„Ci troviamo di fronte a immense difficoltà, se vogliamo dare una definizione concettuale a questo fenomeno che sta più o meno alla periferia della vita reale, e se vogliamo rivelarne la struttura. Questo fenomeno che risulta così leggero nel suo svolgersi, è così solido e impenetrabile per darne una interpretazione concettuale."

(ČERNÝ 1971, p. 151)

Poi Erik Erikson aggiunge con tono scherzoso: „Il gioco - conformemente al suo nome - cerca di tradire ogni definizione", mentre Kolarits considera tutti i tentativi difficoltosi di dare una definizione al gioco come "il gioco scientifico” degli autori. È sorprendente che quasi non si rivela più dell'evidenza del gioco che ,viene giocato sempre per gioco" (SCHEURL). ${ }^{5}$

La tipologia del gioco di Caillois evidenzia la problematica complessa delle caratteristiche convergenti e divergenti delle varie manifestazioni del gioco. Egli in base ai principi dinamici interni stabilisce i quattro tipi più importanti nell'ammasso dei giochi. I fattori dominanti e i nomi dei singoli tipi sono i seguenti: agon (competizione), alea (fortuna), mimikri (imitazione), ilinx (euforia) (CAILlOIS 1967;1981).

I giochi tipici dell'agon sono: il calcio, il biliardo, gli scacchi... L'elemento importante dell'agon è la lotta che viene combattuta con la chance uguale delle parti secondo regole prestabilite. Gli esempi dell'alea sono: i giochi di dadi, la roulette, la lotteria... È caratteristico dell'alea che essendo decisivamente fatale i giocatori non possono influenzare l'esito del gioco. Il domino, la maggior parte dei giochi a carte e molti altri sono delle varianti di un miscuglio dell'agon e dell'alea. Secondo l'osservazione di Caillois mentre gli elementi dell'agon sono reperibili anche nel mondo animale, quelli dell'alea sembrano particolarmente umani. Tra gli esempi del mimikri possiamo elencare il recitare il ruolo di un predone di mare o di Amleto. Il loro elemento essenziale sta nel credere e far credere agli altri di essere qualcun altro ed è evidente che i drammi e gli spettacoli teatrali appartengono a questa categoria del gioco. Gli esempi dell'ilinx possono essere l'altalena, il carosello, i dervisci girovaghi. L'elemento importante di questo tipo del gioco è che i giocatori con il giro veloce o la stramazzata simulano dentro di loro la sensazione dell'euforia e della confusione. I bambini giocano spesso questo tipo di gioco che li fa sentire come se volassero. La classificazione di Caillois stabilendone i quattro tipi dà una sistemazione dei giochi. Ogni tentativo di categorizzare il gioco anche questo - ha i suoi vantaggi, ma nello stesso tempo ha anche i suoi limiti particolari. Bisogna aggiungere alle categorie prima elencate che oltre le varie tipologie del gioco dobbiamo anche definire i segni che uniscono il mondo multiforme dei giochi.

\footnotetext{
${ }^{5}$ Qui le versioni italiane delle citazioni sono state fatte in base alle citazioni ungheresi.
} 
Le forme più concrete del gioco, sono i giochi di società comuni, come nascondino, mafia, ecc., tramite i quali è possibile esaminare anche i giocatori che vi partecipano e a questi giochi sono di solito legate le nostre esperienze personali per via delle quali possiamo conoscere quella "sensazione” che i teorici chiamano „l'esperienza del gioco” e cercano di esplicare metodicamente nelle loro riflessioni

Vediamo ora come si definisce il gioco e quali sono i suoi segni distintivi.Roger Caillois, autore della tipologia sopracitata stabilisce i seguenti criteri per dare una definizione al gioco:

a) attività libera, che il giocatore non può fare contro la sua volontà, e di cui non si può perdere la voglia;

b) attività che si distingue per tempo e spazio proprio;

c) attività di esito vago, senza procedimento o risultato prestabilito;

d) attività improduttiva, che non produce né beni, né ricchezza;

e) attività che dispone delle regole convenzionali;

f) attività fittizia in rapporto speciale con „un'altra realtà” $\mathrm{o}$ in rapporto particolare con la vita quotidiana della „libera non realtà”. (ČERNÝ 1971, p. 142)

In base alle opere di Eugen Fink è possibile proporre una interpretazione esistenziale del fenomeno del gioco la quale stabilisce le seguenti caratteristiche strutturali:

a) il gioco è fonte dei piaceri (sia sensuali che intellettuali);

b) il gioco ha senso (interno ed esterno);

c) il gioco ha carattere sociale (è aperto verso gli altri);

d) si sceglie liberamente, ma si gioca secondo regole obbligatorie per tutti;

e) al gioco appartengono mezzi, con cui si gioca. (Ibid. p. 155)

Infine possiamo citare la definizione, ormai diventata classica che riassume la concezione huizinghiana del gioco:

„Il gioco è un'azione, o un'occupazione volontaria, compiuta entro certi limiti definiti di tempo e di spazio, secondo una regola volontariamente assunta, e che tuttavia impegna in maniera assoluta, che ha un fine in se stessa; accompagnata da un senso di tensione e di gioia, e dalla coscienza di 'essere diversi' dalla 'vita ordinaria'."

(HUIZINGA 1946, p. 49)

Oltre le descrizioni sopracitate di R.Caillois, di E.Fink, e di J.Huizinga, hanno fatto altre importanti osservazioni filosofiche in questione H. G. Gadamer (1986, pp. 177-185), A. Gehlen (1966;1983) e G. Csepregi (1994, pp. 177-188). Secondo loro il gioco è:

a) attività non produttiva che sta per sé stessa avendo in sé stessa il proprio obiettivo;

b) attività vissuta con gioia solenne scaturita dentro noi, un'esistenza senza oneri in cui ci obliamo totalmente;

c) attività che si fa liberamente che allo stesso tempo è sottoposta alle regole stabilite da se stessa ma obbligatorie all'interno del gioco per mezzo delle quali si obbedisce all' ordine del gioco;

d) attività creativa, aperta nel suo svolgimento interno, con fattori liberi;

e) attività che presuppone anche la presenza di un/dei compagno/i di gioco o mezzo/i di gioco nel loro movimento perpetuo;

f) attività isolata nel tempo e nello spazio; 
g) il gioco ha il suo „mondo interno” caratterizzato da tempo e spazio propri, un mondo coerente nella propria realtà,in cui i partecipanti del gioco (i giocatori) entrano volontariamente dal ,mondo esterno";

h) il mondo interno del gioco è caratterizzato dalla „transustanziazione” dei mezzi di gioco, dall'apparizione della posta interna al gioco e dalla „,oscienza di gioco” paradossale dei giocatori;

i) infine, l'esperienza del gioco nella sua forza simbolica offre un',esperienza di esistenza concentrata", aperta ad una certa Totalità, servendoci da modello di esistenza. ${ }^{6}$

\subsection{I tre livelli d'interpretazione del gioco ${ }^{7}$}

Del gioco si parla in più sensi che sono in stretta connessione anche con l'universo, con gli animali e con l'uomo (BRUNER; JOLLY \& SYLVA 1976; 1981abc, 1995) L'interpretazione psicogenetica, biogenetica e antropologico-culturale del gioco umano mette in evidenza un fattore importante. Dennis Palmer Wolf richiama l'attenzione su due importanti caratteristiche delle manifestazioni del gioco: alcune dispongono in gran parte del valore simbolico e altre di caratteristiche fondamentalmente culturali (cfr. SMITH 1984, p. 175). La variabilità dell'approccio del gioco può determinare tre livelli fondamentali d'interpretazione:

I. Al primo livello di interpretazione il gioco viene considerato un evento separato del mondo. In questo caso si tratta del più concreto avvenimento ludico dell'uomo, descritto con la definizione precedente.

II. Il secondo livello d'interpretazione considera il gioco una caratteristica interna dell'uomo. Questa interpretazione ha il contatto più vivo con la simbolica del gioco della storia delle idee e con l'argomento theatrum mundi.

III. In fine esiste un nuovo approccio teorico che conduce al terzo livello d'interpretazione quando il gioco davanti a noi è una realtà ontologica. Tale interpretazione è importante perché dà la chiave d'ingresso alla problematica del Grande Gioco dell'Essere.

Passando dal primo al secondo e al terzo livello d'interpretazione la definizione precedente del gioco (che riguarda un evento separato) perde di validità e ha bisogno di essere generalizzata. Se conformemente alle pretese del secondo e terzo livello d'interpretazione viene revisionata la descrizione del gioco definita in nove punti , si può ottenere i seguenti criteri di una caratteristica ludica, sia interna umana che quella di tutti gli esseri dell'universo:

a) il gioco dal punto di vista dell'obbiettivo ,è̀ una forma di esistenza che si muove in sè”,

b) è una forma di essere solenne, sottomessa ad un certo ordine (interno),

c) è una forma di essere aperta, realizzata con fattori liberi,

d) è una forma di essere che comporta la caratteristica del „moto di qua e di là”,

e) è una forma di essere autorealizzante, in un certo senso „estatica”.

\footnotetext{
${ }^{6}$ Visto che le definizioni rappresentano il filo conduttore della nostra concezione del gioco, gioverà ricordare i criteri sopracitati del gioco. In seguito, partendo da questa definizione applicabile a una concreta situazione del gioco avremo la possibilità di addentrarci nell'astrazione verso una concezione più sintetica che interpreta il gioco come comportamento interno dell'essere umano e tutto ciò ci renderà possibile di cogliere il simbolismo del gioco. Inoltre, questa concezione più sintetica ci permetterà di affrontare la problematica del Gran Gioco dell'Esistenza.

${ }^{7}$ In questo sottocapitolo, basandoci sull'analisi dettagliata indicata nel mio libro pubblicato in lingua ungherese, riassumiamo brevemente le conseguenze per noi importanti dell'attuale - molto ampia - letteratura profana sul gioco (BAGYINSZKI 1998). Ai singoli accenni indichiamo il retroscena letterario in nota in calce alla pagina dove si trovano le spiegazioni del concetto.
} 
Questa descrizione mostra nel modo più comprensibile, generale e laconico il contenuto del gioco che naturalmente ha dei limiti. Di seguito si cercherà di compensare le difficoltà derivanti dal senso traslato delle definizioni in modo tale da estrapolare più dettagliatamente le caratteristiche dei tre livelli d'interpretazione.

\subsubsection{Il gioco come evento separato del mondo}

Questo livello d'interpretazione fu indicato come punto di partenza perché l'esperienza vissuta del gioco si presenta prima di tutto nella forma di un avvenimento concreto del gioco. Tale esperienza è nel centro delle riflessioni del teorico di gioco. Il gioco - in base al primo livello d'interpretazione - è strettamente legato ad uno stato fisiologico ben descrivibile dell'organismo umano (,play-face”, „tono emozionale" ecc.) (GRASTYÁN 1985). L'introspezione soggettiva, il ruolo dell'autocoscienza e la presenza esistenziale nella realtà simbolica del gioco indicano però che la situazione di gioco afferra l'uomo non soltanto fisiologicamente ma anche spiritualmente (BALOGH 1991).

Nel corso dell'interpretazione del gioco umano la letteratura parla del „bilateralismo paradossale”, di un „modo esistenziale misto” e dell',esistenza doppia” (FINK 1960; 1969b). L'espressione "coscienza di gioco" raggruppa il particolare sistema di rapporti con il mondo reale, vissuto dal giocatore il quale entra nel circolo magico del gioco facendo parte dell'ordine solenne di esistenza, in apertura verso la realtà completa del gioco. La coscienza di gioco comprende allo stesso tempo i motivi di esistenza riflessivi e quelli estatici: il giocatore da un lato è costantemente consapevole della situazione di gioco cioè che ,è in gioco", dall'altro canto vive all'interno del mondo del gioco, quasi dimenticandosi di sé stesso nella realtà interna del gioco. Tale struttura paradossale della coscienza del gioco comporta la sensazione di libertà dello staccarsi leggermente dalla realtà quotidiana e dall'altro canto tiene presente la disciplina -volontariamente assunta- delle regole interne del gioco. Il giocatore - con l'espressione di Arnold Gehlen - vive la sensazione particolare della "lotta spogliata di peso" (GEHLEN 1966). Il segreto del gioco sta nel fatto che il mondo interno ed esterno restano in contatto intenso e attraverso i simboli avviene una particolare comunicazione, quindi possiamo pensare che nel gioco ci si apre non solo verso la realtà stessa ma anche verso quella ,assoluta” (cfr. CSEPREGI 1994, pp. 177-188).

Per quanto riguarda le condizioni di possibilità del gioco, Il comportamento giocoso si verifica già al livello biologico quando si è privi di problemi esistenziali e si è in sicurezza relativa. Tali condizioni in caso degli animali si verificano naturalmente nel periodo iniziale di vita , protetto da genitori (cfr. CSÁNYI 1985, pp. 431-435). Nel caso dell'uomo sono importanti l'effetto delle condizioni culturali ed ambientali manifestatesi nell'attività ludica, nonché la connessione tra il tempo libero nella società e tra la sfera dei giochi. La caratteristica ludica è una parte elementare della vita umana, ma allo stesso tempo è fortemente in pericolo perché la lotta di sopravvivenza ed il mondo del consumismo soffocano „l'inutilità” del gioco sia al livello individuale che sociale (cfr. HANKISS 1997, pp. 151174).

\subsubsection{Il gioco come il comportamento interno dell'uomo}

Parlando del comportamento interno giocoso dell'uomo si deve pensare alle manifestazioni esistenziali ed intellettuali del soggetto umano. L'uomo nello stato spirituale del gioco realizza la sua identità homo ludens. Questo vuol dire che con il comportamento interno del gioco l'autocoscienza umana cambia qualifica e passa ad un grado superiore nel vivere la libertà soggettiva e nell' assumere l'autoidentificazione umana. Nell'atteggiamento dell'uomo intelligente la creatività contemplativa del gioco è uno dei segni importanti dell'orientamento mentale.

L'importanza delle attitudini interne ludiche non si limita soltanto al campo della vita intellettuale ma è valida anche in una connessione molto più ampia. In genere si può dire che la nostra vita si 
delinea nel dialogo tra le circostanze esterne e le risposte o reazioni interne date ad esse (MILLAR 1968). Sebbene qualche volta non possiamo cambiare le circostanze esterne, il nostro mondo interno spirituale con il suo ordine di valori può essere capace di trasformare il valore negativo o positivo dei fatti. La nostra vita quindi può avere due aspetti ben distinti: la lotta per la nostra esistenza oggettiva nella società e l'esperienza interna, soggettiva ed esistenziale di essa. La manifestazione delle attitudini interne ludiche significa che la sfera interna soggettiva ed esistenziale è molto indipendente dalla sfera del mondo esterno crudele. In questo modo l'uomo può avere la capacità di dominare in un certo senso, senza potere oggettivo, i fatti della realtà. Queste intuizioni sono in relazione alle esperienze degli psicoterapeuti: secondo loro il metodo della terapia consiste nel portare il paziente dallo stato non caratterizzato dal gioco allo stato d'animo del gioco (cfr. WiNNICOTT 1997, p. 79). Seguendo le idee di Erik Erikson l'importanza dell'attitudine interna ludica può essere descritta come un campo sottile che si apre nell'anima dell'uomo diventato un'altra dimensione indipendente dallo spazio, dal tempo, dalla causalità e dalle necessità sociali.

\subsubsection{Il gioco come realtà ontologica}

Parlando del gioco degli animali oppure del mondo materiale ${ }^{8}$ si deve passare al terzo livello dell'interpretazione del gioco. I tentativi di elaborare l'argomento sul Grande Gioco dell'Essere non equivalgono soltanto ad un allargamento sopradimensionato della metafora-gioco? Gli scienziati e i filosofi dimostrano che dobbiamo considerare la possibilità di interpretare il gioco nel modo sorprendentemente globale. ${ }^{9}$ Tra le teorie filosofiche sul gioco „l'ontologia del gioco" di Gadamer analizza il modo di essere delle opere d'arte per vedere il gioco dall'ottica orientata all'oggetto, di essere separato dal soggetto umano (GADAMER 1960; 1983).

Manfred Eigen, premio Nobel, ed il suo collega autore (cfr. EIGEN \& WINKLER 1986, p. 10), riflettendo sulle esperienze metaempiriche delle scienze della natura interpretano la completezza dinamica dell'Universo come metamorfosi complessa del gioco. Tutto quello che succede nel nostro mondo è la manifestazione della realtà conseguente (cioè realtà che fa valere le sue regole interne) per l'uomo. Queste sono le regole interne e i formulari della „,natura cosmica" che non costituiscono lo spazio fatalmente determinato che si chiude in sé, ma - secondo la meccanica quantistica - restano aperte ai fattori formativi della casualità. Cosí, la facciata meta-empirica della realtà non indossa i costumi dominanti della necessità rigorosa oppure della spontaneità imprevedibile delle epoche passate, ma porta le caratteristiche dell'attitudine ludica che nasce nella dicotomia della spontaneità e della fatalità. L'attitudine ludica della nostra immaginazione meta-empirica sulla realtà, manifestatasi nel gioco d'insieme delle regole e della fatalità, viene presentata da Eigen. L'intero libro di Eigen mette in evidenza che la priorità spetta al terzo livello d'interpretazione nella definizione del gioco:

\section{„Il gioco lo consideriamo un fenomeno della natura che è la base di tutte le azioni nella sua dicotomia derivante dalla casualità e fatalità."}

Nell'approccio di Gadamer ed Eigen il gioco equivale al principio di esistenza dinamico della natura cosmica. Su questo orizzonte della teoria del gioco tutte le manifestazioni precedentemente descritte del gioco possono essere interpretate come singole rivelazioni metamorfiche del principio di

\footnotetext{
${ }^{8}$ È una questione importante quella su cui ci chiediamo in quale senso possiamo parlare (se in fondo è possibile parlarne in questo caso) del gioco o dell'attività di gioco dal punto di vista degli esseri privi di coscienza e di vita.

9 È interessante anche il fatto che il terzo livello d'interpretazione non significa un'astrazione molto lontana dall'intuizione linguistica, visto che la lingua e la simbolica legata al gioco riesce a comprendere senza problemi le affermazioni grandiose relative al gioco della natura.
} 
esistenza. Anche il secondo ed il terzo livello d'interpretazione sono basati sul terzo dal punto di vista ontologico. ${ }^{10}$

\section{La complementarità: l'antropologia e la teologia del gioco}

Le opere di Johann Huizinga e di Hugo Rahner le quali portano lo stesso titolo (Homo ludens) in merito al concetto del gioco rivestono una notevole importanza per la letteratura teologica nonché per le scienze profane.

\subsection{Il „dialogo” di Hugo Rahner con Johann Huizinga}

Il libro di Huizinga esercitò una notevole importanza culturale per il tema qui trattato. L'eminente studioso stupì non poco quando nel 1933 pronunciò come prolusione all'università di Leyda - di cui era anche rettore - la lezione sul gioco che cinque anni dopo divenne un libro con il titolo, da allora proverbiale, di Homo ludens (HUIZINGA 1938;1946). La tematica del gioco ebbe da quel momento, come si è accennato, la sua summa, da cui non si poté più prescindere. Anche se prima di allora c'era un certo interesse per il gioco (per fare due esempi molto differenziati basti pensare a Schiller o agli psicologi sperimentali) Huizinga con un balzo porta la tematica su un altro livello, tutt'oggi rilevante, quello del discorso filosofico e della storia delle idee, inserendolo tuttavia concretamente in ogni attività umana, quale momento genetico della cultura. Isolandolo lo concretizza, focalizzandolo e considerandolo come invariante culturale lo immerge nella realtà storica, sociale, antropologica e sociologica. Se il gioco è una forma o uno schema, la sua individuazione rende tuttavia intellegibile l'epoca nella sua singolarità e nella sua irrepetibilità.

La novità di un tale approccio non poteva che destare scalpore e il libro divenne subito un caso, piuttosto nella cultura italiana, ma anche altrove e Umberto Eco nella prefazione sottolinea giustamente come il Homo ludens avesse molte qualità per provocare il lettore di quegli anni:

\section{„Un impudente gusto interdisciplinare, una liberale curiosità per le culture non europee, uno spregiudicato coraggio nel livellare i portati della cultura alta alle manifestazioni quotidiane della vita."}

Ma ancora Eco ricorda altresì che i concetti per noi oggi accettati e consolidati quali la cultura come complesso di saperi anche minori, o di non saperi (sport), e la nozione di invariante culturale, tipica dell'antropologia culturale ma ostica alla filosofia idealistica sono una novità per il lettore degli anni '50. Si può concludere dicendo che quelle critiche sono forse l'aspetto di maggiore significanza per noi oggi, come pure l'osservazione di Eco secondo cui manca in Homo ludens la sistematizzazione in schemi e formule del concetto di gioco. Infatti, le definizioni più esatte del termine ,gioco” sono state elaborate proprio dai critici dell'opera di Huizinga.

\subsection{Il motivo del uomo giocante da Johann Huizinga}

Nel gioco abbiamo a che fare con una categoria di vita assolutamente primaria, facilmente riconoscibile da ognuno, con una sua ,totalità”. Johann Huizinga definisce il gioco così:

„Gioco è un'azione, o un'occupazione volontaria, compiuta entro certi limiti definiti di tempo e di spazio, secondo una regola volontariamente assunta, e che tuttavia impegna in maniera assoluta, che ha un fine in se stessa;

\footnotetext{
${ }^{10}$ L'estrapolazione concettuale riguardante i tre livelli della simbolica del gioco può essere considerato come quadro filosofico delle osservazioni analitiche conseguenti sui punti di collegamento tra la simbolica del gioco e la teologia.
} 


\section{accompagnata da un senso di tensione e di gioia, e dalla coscienza di 'essere diversi' dalla 'vita ordinaria'."}

(HUIZINGA 1946, p. 49)

Tutti i popoli giocano anzi giocano in modo curiosamente simile ma non ne consegue affatto che tutte le lingue racchiudano l'idea del gioco in un'unica parola con tanta sicurezza e anche con tanta larghezza come fanno le lingue moderne europee. Non è forse mero caso che proprio alcuni popoli che in svariate forme hanno avuto sempre nel sangue il gioco nondimeno distribuiscono l'espressione di tale attività in molte parole diverse (il greco, il sanscrito, il cinese) (cfr. ibid., p. 51.). Tutti i termini di questo gruppo di nozioni a malapena coerenti a cui appartengono il gioco, il riso, la facezia, lo scherzo, il comico e la follia, hanno in comune quell'irriducibilità di concetto che dovremmo assegnare al gioco. La loro ratio sta in uno strato particolarmente profondo del nostro essere spirituale.

\subsubsection{Il gioco preumano}

Il gioco è più antico della cultura, perché il concetto di cultura, per quanto possa essere insufficientemente definito, presuppone in ogni modo la convivenza umana. Gli animali giocano proprio come gli uomini; tutte le caratteristiche fondamentali del gioco sono realizzate in quello degli animali. Basta osservare i cuccioli nel loro gioco per scorgere in quell'allegro ruzzare tutti questi tratti fondamentali. Curioso è inoltre il fatto che proprio gli uccelli filogeneticamente tanto distanti dall'uomo ciononostante hanno tante cose in comune con lui: i fagiani di montagna eseguono danze, le cornacchie tengono gare di volo, gli uccelli del paradiso e altri adornano i loro nidi, gli uccelli cantatori eseguono melodie. La realtà ,gioco”, percepibile da ognuno, si estende sopra l'insieme del mondo animale e umano (cfr. ibid., p. 50).

\subsubsection{Il gioco spirituale}

Già nelle sue forme più semplici, il gioco umano è qualche cosa superiore di un fenomeno puramente fisiologico e di una reazione psichica fisiologicamente determinata (cfr. ibid., p. 17). Il gioco come tale oltrepassa i limiti dell'attività puramente biologica cioè è una funzione contenente un senso. Al gioco partecipa qualcosa che oltrepassa l'immediato istinto a mantenere la vita, e che dà un senso all'azione del giocare.
„Ogni gioco significa qualche cosa. Se chiamiamo spirituale questo principio attivo che dà al gioco la sua essenza, allora diciamo troppo; se lo chiamiamo istinto non diciamo nulla. Comunque lo si consideri, certamente si manifesta, con tale 'intenzione' del gioco, un elemento immateriale nella sua essenza stessa." (Ibid., p. 19)

L'intensità del gioco non è spiegata da nessuna analisi biologica, eppure in quell'intensità, in quella facoltà di far delirare sta la sua essenza, la sua qualità. La natura logicamente avrebbe potuto dare alla sua prole tutte quelle funzioni utili di scarico di energia, di rilassamento, di preparazione e di compenso anche nella forma di esercizi e reazioni puramente meccanici. Invece no, ci diede il gioco con la sua tensione, con la sua gioia, col suo „scherzo". Insieme al gioco però si riconosce anche volente o nolente lo spirito. Perché il gioco, qualunque sia la sua essenza, non è materia visto che oltrepassa già nel mondo animale i limiti dell'esistenza fisica.

L'uomo che volge lo sguardo alla funzione del gioco, non nella vita animale, né nella vita puerile, ma nella cultura, ha il diritto di impadronirsi del gioco là dove la biologia e la psicologia lo trascurano. Egli trova il gioco nella cultura come una data grandezza, esistente prima della cultura stessa che ne viene accompagnata e poi attraversata dal principio sino alla fase di cultura in cui l'indagatore stesso vive. Egli trova dappertutto presente il gioco come un proprio modo d'agire che si distingue dalla vita „ordinaria”. Le grandi attività originali della società umana sono tutte già intessute di gioco. 
L'inferiorità del gioco ha i suoi limiti nella superiorità della serietà. Il gioco si converte in serietà, la serietà in gioco. Il gioco sa innalzarsi a vette di bellezza e di santità che la serietà non raggiunge (cfr. ibid., p. 25).

Alle sacre rappresentazioni nel culto delle civiltà arcaiche, in confronto col gioco infantile partecipa un elemento spirituale di più, difficilissimo da definire con precisione. La sacra rappresentazione è qualche cosa di più di una realizzazione apparente e magari simbolica: è una realizzazione mistica. Nella rappresentazione si concreta qualche cosa d'invisibile, d'inesprimibile in forma bella, reale e sacra. I partecipanti al culto sono convinti che l'azione realizzi una certa salvezza e promuova uno stato di cose superiore a quello in cui essi vivono ordinariamente. Nella forma e nella funzione d'un gioco (che è una qualità indipendente del vivere) l'idea di esser compresi in un cosmo, cioè in un ordine sacro, ottiene la sua primeva e suprema espressione. Dentro il gioco viene incuneandosi a mano a mano il senso di un ,atto" sacro. Il culto s'innesta nel gioco però il giocare in sé fu il fatto primario e il carattere ludico può essere inerente alle azioni più elevate.

„Si potrebbe dunque andare avanti con questa successione sino alle pratiche del culto, attestando che anche il sacerdote che compie gli atti rituali non fa che giocare un gioco?" (Ibid., p. 37)

- chiede Johann Huizinga. Le domande che sorgono a questo punto toccano il fondo della nostra coscienza.

\subsubsection{Gli elementi ludici della cultura}

„Parlando dell'elemento ludico della cultura, non intendiamo dire che fra le varie attività della vita culturale $i$ giochi occupino un posto importante, $e$ neppure che la cultura provenga dal gioco per un processo di evoluzione, di modo che ciò che in origine era gioco sia passato più tardi in qualcosa che non sia più gioco e che possa portare il nome di cultura. La concezione chiarita qui sotto è la seguente: la cultura sorge in forma ludica, la cultura è dapprima giocata. Anche quelle attività che sono indirizzate alla soddisfazione dei bisogni vitali, come per esempio la caccia, nella società arcaica assumono di preferenza la forma ludica."

(Ibid., p. 69)

In tale „dualità-unità” di cultura e gioco, il gioco è il fatto primario, oggettivo, percepibile, determinato concretamente mentre la cultura non è che la qualifica applicata dal nostro giudizio storico al dato caso.

La rivalità sotto forma di gioco come fautore di vita sociale più antica di qualsiasi cultura stessa dominò il vivere umano sin dai primordi e maturò come un fermento le forme della cultura arcaica. Il culto sorse e crebbe in gioco sacro. La poesia nacque in gioco e continuò a vivere di forme ludiche. Musica e danza erano gioco puro. Saggezza e sapere si manifestarono in gare sacre. Il diritto dovette svincolarsi dal gioco sociale. Il regolamento della lotta con le armi e le convenzioni della vita nobile erano basati su forme di gioco. Si ricavò la logica conclusione che la cultura nelle sue fasi originarie viene giocata. La cultura non nasce dal gioco come frutto vivo che si svincoli dal corpo materno, ma si sviluppa nel gioco e come gioco.

Dacché esistono parole indicanti la lotta e il gioco, si è dato il nome di gioco anche al combattere. Il combattere, essendo funzione culturale, presuppone sempre delle regole limitanti, ed esige in una certa misura il riconoscimento di una qualità ludica (ibid., Capitolo V). Ogni combattere legato a regole limitanti ha proprio per quell'ordine limitante il carattere formale di un gioco, anzi della forma di gioco più intensa, più energica, e nello stesso tempo più evidente. Il lecito nel gioco non sempre s'arresta al versamento di sangue e neppure all'omicidio. Il torneo medievale fu indubbiamente un 
combattimento simulato dunque un gioco eppure nelle sue primissime forme deve essere stato completamente e manifestamente ,tutta serietà" fino alla morte. Tutto ciò che ora vediamo come un gioco nobile e bello, una volta è stato un gioco sacro. L'iniziazione a cavaliere, il torneo, l'ordine e il voto hanno senza alcun dubbio le loro origini nelle cerimonie d'iniziazione di tempi primordiali. Su tali restrizioni era fondato fino a tempi recenti il diritto internazionale il quale esprimeva l'aspirazione degli uomini a contenere la guerra nella sfera della civiltà. Lo stato di guerra era nettamente distinto dallo stato di pace da un lato e dalla violenza criminale dall'altro. Al di sopra dei partiti aleggiava l'idea di una collettività che riconosceva come „umanità" i propri membri reclamanti il diritto a essere trattati come uomini. È stata la teoria della „guerra totale” ad abbandonare gli ultimi resti della funzione culturale della guerra e dunque della sua funzione ludica.

A prima vista la sfera del diritto, della legge e della giustizia è lontanissima da quella del gioco. Infatti tutto ciò che è diritto e giustizia viene dominato da sacrosanta serietà e dall'interesse vitale dell'individuo e della sua collettività. Una certa affinità fra diritto e gioco ci si rivela chiaramente non appena si nota che la pratica del diritto possiede al massimo grado il carattere competitivo. L'effettivo rapporto fra diritto e gioco nelle culture arcaiche è classificabile da tre punti di vista diversi (ibid., Capitolo IV). Il processo è un gioco d'azzardo, è una gara, è una lotta a parole. Pur condotto talvolta sino in fondo il duello giudiziario ha fin dall'inizio la tendenza a rilevare i suoi lati formali accentuando con ciò i suoi tratti ludici. Già la possibilità di farlo eseguire da un campione assoldato proviene dal suo carattere rituale; infatti proprio il tono di sacramento di un'azione permette in genere una sostituzione. Tanto la qualità ludica come quella competitiva, ambedue risolte in quella della sacralità che ogni gruppo richiede per la sua giustizia, traspaiono ancor oggi in molte forme della vita giuridica. Tuttora i giudici escono dalla ,vita solita” prima di cominciare il lavoro giudiziario. Si vestono della toga oppure si mettono la parrucca. Ogni luogo di giustizia è un vero temenos, un sacro luogo isolato, tagliato fuori per così dire dal mondo delle cose consuete.

La personificazione di astrazioni di solito viene considerata come un tardo prodotto d'invenzione di scuola. Ci sono buone ragioni per supporre che tale personificazione di diverse qualità sia piuttosto una delle funzioni primordiali della formazione religiosa, quando le forze e le potenze di cui l'uomo si sentiva circondato non avevano ancora assunto aspetto umano (cfr. ibid., p. 175). La mente, prima ancora di concepire figure divine in forma umana, tutta commossa e trascinata dalle cose misteriose ed enormi con cui la natura e la vita provocano, comincia a dare nomi alle cose che l'opprimono o elevano e tali cose sono vedute dalla mente come esseri ma non ancora o a malapena come figure. Per tutti quei casi è lecito domandare se questo personificare sorga da o risulti in un'attitudine spirituale a cui si possa dare il nome di fede sincera. Non rappresentano invece tutte quelle personificazioni un gioco dello spirito, da capo a fondo (ibid., Capitolo VIII)? Esempi tolti da un periodo più recente fanno propendere per tale conclusione. San Francesco d'Assisi adora la sua sposa Povertà col più sacro fervore, in pia estasi. Ma qualora si avanzasse la fredda domanda se egli avesse creduto a un essere trascendente, a un essere celeste chiamato Povertà, a un essere dunque che veramente era l'idea della Povertà, la risposta non può che incagliarsi. Già nel formulare la domanda in tali termini freddamente logici venne forzato il sentimento inerente all'immagine. Francesco ci credeva e non ci credeva. La Chiesa lo autorizzava a malapena, e almeno non espressamente, a una tale fede. Lo stato d'animo con cui concepiva la Povertà doveva restare in equilibrio fra l'immaginazione poetica e il dogma confessato seppure tendesse verso quest'ultimo. L'espressione più calzante per tale attività spirituale è ancora questa: Francesco giocava con la figura della Povertà. E tutta la vita del Santo d'Assisi è davvero piena di figure e fattori puramente ludici i quali gli danno il suo aspetto più bello e attraente (cfr. ibid., p. 176). Ma l'ambiente ludico ove giocano santi e mistici sta al di sopra della sfera del pensiero logico, cioè è una sfera inaccessibile a una speculazione che dipende dalle capacitá logiche. Le nozioni di gioco e di santità si rasentano sempre; come si rasentano l'immaginazione 
poetica e la fede. Se la tendenza sempre attiva dello spirito a vedere come persone le cose con cui l'uomo ha rapporti nella vita è davvero radicata nel contegno ludico, allora si fa avanti una questione di primaria importanza se il contegno ludico debba essere esistito prima che vi fosse cultura umana o facoltà di parola e d'espressione.

Tutti gli elementi e i mezzi della poesia possono essere intesi nel miglior modo come funzioni ludiche (ibid., Capitolo VII). L'elemento ludico e ogni forma di attività poetica sono tanto strettamente connessi alla natura della poesia che si hanno tanti punti di contatto con la struttura del gioco e questo intimo rapporto sembra ormai indissolubile. Nella tragedia e nella commedia la provenienza dal gioco si manifesta continuamente e anche la lingua rispecchia tale stretto legame, in particolare il latino e le lingue che hanno attinto alla fonte del Latium, dove il dramma è chiamato un gioco. Resta però un altro fatto che la lingua greca non abbia concepito una parola universale per l'intero dominio del gioco ed è curioso che proprio presso i greci che crearono il dramma nella sua forma più perfetta la parola per gioco non sia applicata alla rappresentazione teatrale o allo spettacolo stesso. In un certo senso la società ellenica era in tutte le sue manifestazioni così profondamente imbevuta dell'elemento ludico che questo riaffiorava ormai a malapena alla coscienza come cosa eccezionale. Tra i grandi generi distinti in poesia secondo l'immortale esempio greco il genere lirico è quello maggiormente situato nell'originaria sfera del gioco. L'elemento lirico è il più distante dall'elemento logico e il più vicino alla danza e alla musica. La funzione fortemente liturgica e sociale della parola poetica nelle culture arcaiche è connessa intimamente al fatto che il proferire parola era in quel periodo ancora inseparabile dalla recita musicale. Ogni culto vero viene cantato, danzato, giocato. Null'altro è tanto adatta a compenetrare noi, portatori di tarda cultura, della nozione di gioco sacro quanto la emozione musicale. La sensazione estetica e quella sacra si fondono nel godimento della musica anche senza riferirsi a concetti religiosi formulati, e in tale fusione svanisce ogni contrasto gioco-serietà. La vera indole di ogni attività musicale è un giocare. In fondo rimane pur sempre gioco se la musica serva al sollazzo e alla gioia o che voglia esprimere profonda bellezza o che abbia una sacra destinazione liturgica. Proprio nel culto essa è spesso unita intimamente a quella funzione ludica per eccellenza: la danza. Il rapporto fra la danza e il gioco non è quello di un ,partecipare a”, ma di un „far parte di”, è dunque il rapporto di un'essenziale identità. La danza è una forma speciale e perfetta del giocare stesso come tale.

Volgendoci dalla poesia, dalla musica e dalla danza al campo dell'arte figurativa, ivi i rapporti col gioco sono assai meno evidenti (ibid., Capitolo X). L'architetto, lo scultore, il pittore o il disegnatore, l'artista vasaio e in generale l'artista decorativo, con un lungo e diligente lavoro fissa il suo impulso estetico nella materia e una volta prodotta essa esercita, immobile e muta, la sua azione fin tanto che ci sarà gente che amerà guardarla. Quando viene a mancare un'azione pubblica nella quale l'opera d'arte si fa viva ed è goduta, sembra che nel dominio delle arti plastiche o figurative non ci sia più posto in fondo per un fattore ludico ma nonostante questo contrasto fondamentale è possibile indicare in diversi punti pure nell'arte figurativa il fattore ludico. Una relazione fra arte e gioco è stata ammessa già da lungo tempo in quella teoria che voleva spiegare la creazione di una forma d'arte a partire dall'impulso innato verso il gioco. Infatti non si può misconoscere un quasi istintivo e spontaneo bisogno umano di ornare che a ragione può essere chiamata una funzione ludica. Anche assegnando un significato tanto primordiale e essenziale al gioco come fattore di cultura, non si considerano risolte le origini dell'arte riferendole all'innato istinto ludico sebbene sia difficile davanti a molti prodotti della sovrabbondante ricchezza delle arti figurative reprimere l'idea di un gioco della fantasia, di un „giocoso e giocante" creare dello spirito e della mano.

La filosofia è l' antico gioco dell'ingegno che nelle culture arcaiche - già nei periodi più primordiali - passa continuamente e insensibilmente dall'atto sacro al mero divertimento: un gioco che ora sfiora la massima saggezza, ora è solamente scherzosa rivalità (ibid., Capitolo IX). I due motori essenziali di 
gioco sociale, la rappresentazione di un essere strano e la vincita pubblica del rivale sono evidentissimi nella funzione del sofista greco. Egli si offre di parlare di qualunque soggetto preparato appositamente per esporlo e di rispondere a qualunque domanda che gli si voglia fare. Insomma pretende di non aver mai incontrato il suo superiore. Si tratta d'un grande avvenimento quando giunge in città un sofista famoso perché i sofisti sono ammirati come taumaturghi e paragonati ai lottatori - insomma la pratica dei sofisti si svolge completamente nella sfera dello sport quando gli spettatori acclamano e ridono a un frizzo riuscito. È un vero e proprio gioco: i sofisti avvincono l'avversario come in una rete oratoria, lo mettono knock-out, si vantano di fare soltanto domande insidiose a cui la risposta non può che riuscire sbagliata sempre. I sofisti stessi si sono resi conto benissimo del carattere ludico della loro attività. Il sofisma sfiora il comune indovinello inteso come divertimento ma allo stesso tempo anche il sacro enigma cosmogonico.

All'origine di ogni competizione c'è il gioco, che è una convenzione: quella di compiere entro un limite di tempo e di spazio, secondo date regole, in una data forma, qualche cosa che sciolga una tensione e che esorbiti dal corso normale della vita. Quello che si debba compiere e quello che si ottenga con ciò è una questione che si collega solo in secondo luogo alla funzione del gioco. Una straordinaria omogeneità distingue in tutte le civiltà le usanze competitive e il significato ascrittovi e quest'uniformità quasi perfetta dimostra da sola come tutta l'attività ludica-agonale sia ancorata profondamente nell' anima e nel vivere sociale degli uomini (ibid., Capitolo III). Nel campo giuridico e agonale l'uniformità della cultura arcaica si manifesta nelle competizioni di scienza e saggezza. Per l'uomo primitivo il potere e l'osare qualche cosa significa forza, ma il sapere qualche cosa significa forza magica e in fondo ritiene ogni conoscenza non comune una scienza sacra, un segreto e magico sapere. Le gare di scienza sacra sono profondamente radicate nel culto e ne formano un elemento essenziale: le domande che i preti sacrificatori si pongono a vicenda dopo sfida, sono degli indovinelli nel vero senso della parola, assolutamente identici per forma e tendenza all'indovinello come gioco di società. L'indovinello è per norma e all'inizio un gioco sacro, cioè trapassa i limiti di gioco e serietà è di massima importanza ma non per questo perde il suo carattere ludico. Se poi l'enigma si dirama tanto verso il dominio del divertimento quanto verso quello della sacra dottrina, non si deve parlare né di serietà che si perde in scherzo né di gioco che si sublima in serietà. È invece la vita culturale che qui produce via via una certa separazione fra i due domini che vengono distinti oggi come serietà e gioco i quali però in una fase primitiva formavano un indiviso medium spirituale in grazia del quale sorse la cultura (cfr. ibid., p. 145).

Si tratta di ben più che di un confronto retorico quando si crede di poter considerare la cultura $s u b$ specie ludi (cfr. ibid., p. 22). La cultura vera non può esistere senza una certa qualità ludica perché la cultura suppone autolimitazione e autodominio, una certa facoltà a non vedere nelle proprie tendenze la mira ultima e più alta, ma a vedersi racchiusa entro limiti che essa stessa liberamente si è imposta. La cultura vuole tuttora, in un certo senso, essere giocata in seguito ad un comune accordo, secondo regole prestabilite. La cultura vera esige sempre e per ogni rispetto fair play, e il fair play non è altra cosa che l'equivalente di buona fede espresso in termini di gioco,. Il „guasta-gioco” guasta la cultura stessa. Se questa qualità ludica vorrà creare o promuovere la cultura, dovrà essere pura non consistendo nel pervertimento o nell'abbandono delle norme prescritte dalla ragione, dall'umanità e dalla fede. Non dovrà essere neanche una falsa apparenza dietro la quale si mascheri un disegno di realizzare date mire con forme ludiche appositamente coltivate. Il vero gioco esclude ogni propaganda e ha in sé la sua finalità.

\subsubsection{Tutto è vanità?}

Alla luce delle cose già menzionate all'antico „tutto è vanità" pare voglia sostituirsi con senso forse più convincente e positivo un: „tutto è gioco”. Sembra una facile metafora, mera impotenza dello 
spirito eppure è la stessa saggezza a cui era giunto Platone quando chiamò l'uomo un giocattolostrumento degli dei. Lo stesso pensiero, in figura strana, si trova nel Libro dei Proverbi. Qui l'Eterna Saggezza, fonte di giustizia e di dominio, dice che prima di ogni creazione essa stava giocando al cospetto di Dio per suo divertimento e che nel mondo della terra essa va giocando i suoi divertimenti con gli uomini $(\operatorname{Pr} 8,30-31)$.

\begin{abstract}
„Il gioco in sé, come dicemmo già all'inizio, è situato fuori della sfera delle norme morali. In sé il gioco non è né buono né cattivo. Quando tuttavia l'uomo deve decidere se un'azione, a cui la volontà lo guida, gli è prescritta come serietà oppure gli è permessa come gioco, allora la sua coscienza morale gli offrirà subito la pietra di paragone. Non appena, sul punto d'agire, nella nostra decisione, intervengono modi di verità o di giustizia, di pietà o di perdono, il problema non ha piú senso. Una sola goccia di compassione basta a elevare le nostre azioni al di sopra delle distinzioni dello spirito pensante. In ogni coscienza morale fondata sul riconoscimento di giustizia e di grazia divina, la domanda: gioco o serietà, che fino all'ultimo rimase insolubile si riduce a tacere per sempre" (ibid., p. 263)
\end{abstract}

- con queste parole conclude Johann Huizinga la sua opera.

\title{
2.3. Il motivo di Dio giocante da Hugo Rahner ${ }^{11}$
}

Hugo Rahner ${ }^{12}$ scrive nella prefazione del suo libro dedicato alla teologia del gioco:

„Ci muoveremo dal punto in cui è giunto, nell'ultima pagina del suo Homo ludens, quel grande interprete della cultura che risponde al nome di Huizinga, dove egli mostra appunto che la prefazione ideale dell'etica umana è una misteriosa riproduzione di quell'eterna Sapienza che gioca dal principio al cospetto di Dio. Di qui prendiamo le mosse noi per tentare una interpretazione teologica e religiosa del gioco. Attingendo agli obliati tesori degli antichi e della Chiesa dei tempi passati vogliamo mostrare che cosa ha perduto l'uomo d'oggi, che non sa più giocare perché è divenuto 'amisterico'. [...] Quando diciamo che 'l'uomo che gioca' è (oppure, purtroppo, era) l'uomo in cui si

\footnotetext{
${ }^{11}$ In questo capitolo, seguendo il saggio quadripartito di Hugo Rahner (Deus ludens - Homo ludens - Ecclesia ludens - La danza celeste), riassumiamo le sue osservazioni colte dall'analisi della tradizione patristica e del sottofondo letterario ellenistico. L'opera del nostro autore cita, tra l'altro, i classici seguenti: S.Agostino, Alonso Rodriguez, S.Atanasio, S. Basilio, Beda Venerabile, Cassiano, S. Cirillo d'Alessandria, S. Clemente d'Alessandria, Meister Eckhart, Eusebio di Cesarea, Filone d'Alessandria, S. Francesco di Sales, S. Gerolamo, S. Gregorio di Nazianzo, S. Gregorio di Nissa, Ippolito, Massimo Confessore, Matilde di Magdeburgo, Balbuziente Notker di S. Gallo, Origene, Prudenzio, Salonio, Sulpicio Severo, S. Teresa di Lisieux, Tertulliano, S. Tommaso d'Aquino, Ugo di S. Vittore, Venturino da Bergamo.

${ }^{12}$ Hugo Rahner nasce a Pfullendorf il 3 maggio 1900 e muore a Monaco il 21 dicembre 1968. Fratello maggiore di Karl, dopo gli studi ginnasiali, nel 1919 entrò nel noviziato della provincia tedesca della Compagnia di Gesù e l'anno successivo passò a Valkenburg, in Olanda, per compiervi gli studi di filosofia. Nel 1926 iniziò gli studi teologici a Innsbruck. Ordinato sacerdote nel 1929, nel 1931 divenne dottore in teologia con una tesi di laurea intitolata Fons Vitae, sulla storia della devozione al Cristo nei primi tre secoli. Nel 1935 conseguì la libera docenza per storia della Chiesa antica e patrologia presso la facoltà teologica di Innsbruck. Dal 1938 al 1945 proseguì la sua attività in Svizzera, partecipando ai convegni che il circolo Eranos organizzava annualmente ad Ascona. Frutto dei suoi interventi è la riflessione teologica sul libro di Huizinga. Il tema di questo libretto di Hugo Rahner (L'homo ludens) fu oggetto di una relazione al congresso „Eranos” tenutosi in Ascona nel 1948, e venne poi pubblicato nel 1949 (RAHNER 1952; 1969). Hugo Rahner tratta del segreto della saggezza umana e cristiana che si concretizza nell'homo ludens. Muovendosi dal punto in cui è giunto Huizinga - nell'ultima pagina del suo Homo ludens -, Rahner tenta una interpretazione teologica e religiosa del gioco.
} 


\section{dispiega la massima cultura, sottintendiamo una visione totale di ciò che} definiamo gioco."

(RAHNER 1969, pp. 10-11)

I putti dell'arte barocca che tanto lietamente giocano coi globi ne sono un ultimo profondo adombramento, attinto dall'abissale verità cristiana che solo „bambini” entrano nel regno dei cieli. Gioco e danza - a patto che quaggiù si realizzino autenticamente e seriamente - sono perciò una anticipazione del cielo; nel gesto o nel suono o nella parola, un'anticipazione di quell'armonia tra anima e corpo rivolti a Dio, che noi chiamiamo cielo. L'eternità sarà dunque quel che fu il perduto paradiso: un divin gioco da fanciulli, una danza dello spirito, una realizzazione infinita ed eterna della corporizzazione dell' anima.

Si pone la domanda quale sia il punto di partenza dell'approfondimento concettuale del termine gioco. Il gioco è anzitutto un' attività umana essenzialmente psico-fisica: la raggiunta espressione di un intimo potere dell'anima coadiuvato dal gesto fisicamente visibile, dal tono percepibile, dalla materia tangibile (cfr. ibid., p. 12). Pertanto l'attività ludica è quell',,alludere" dello spirito alla propria corporizzazione che biologicamente è esercizio corporizzante ben strutturato e psichicamente s'identifica con l'arte nel senso più ampio del termine. Di gioco parliamo poi se questo dominio del corporeo da parte dello spirito ha in qualche modo trovato la propria perfezione nell'agile levità, nella quasi aerea eleganza del ,esser capaci di fare”; se la parola o il suono o il gesto sono disponibili e arrendevoli allo spirito; se il corporeamente visibile è divenuto l'espressione di un'interiore ricchezza paga di sé. Perciò il gioco è un'attività per l'attività, ricca di significato, che ha in sé il proprio scopo. Il bambino che gioca beato, l'artista che suona il proprio strumento, il genio a cui tutto balza di mano come per gioco sono realizzazioni di un' ansia umana primigenia verso una libera, alata e non inibita armonia tra anima e corpo (cfr. ibid., p. 12). Ecco perché il gioco nei suoi primordi fu anzitutto santo, sacro al Divino e la danza fu un atto essenzialmente culturale. E d'altro canto: ecco perché l'uomo che gioca nella sua serena libertà, nella grazia del suo sorridente rifiuto del mondo, nel suo sapiente abbandono, può essere tutto ciò solo se non stravolge il mondo a pura realtà cosmica o psichica ma resta aperto a Dio, solo dunque se ha già compiuto per sé e per la propria forma vitale l'anticipazione dell'eterno, se quasi con l'aerea eleganza del danzatore spinge lontano da sé il globo terrestre e tuttavia stringe al proprio cuore il mondo, epifania trasparente del Dio Creatore.

Il mistero dell'homo ludens può essere colto parlando anzitutto e in profondo - sulla scia di Hugo Rahner - del Deus ludens, del Dio Creatore, di Colui che ha chiamato all'esistenza il mondo degli atomi e degli spiriti come per un immane gioco... poiché anche il più geniale gesto dell'uomo che gioca non è che una infantile e maldestra imitazione del Logos, che ab aeterno „gioca” dinanzi al volto del Padre. Il gioco dell'uomo può essere percepito come una specie di psicografia dell'homo ludens, una descrizione di quel comportamento quasi librato tra serenità e serietà, tra scherzo e tragicità, che i greci definirono inimitabilmente con la formula „uomo serio-sereno". Quest'uomo è in grado di fare della propria vita un bel gioco perché sa che proprio questa vita è tragedia o commedia (,,Scherzo e serietà sono fratelli”, dice Platone nella lettera sesta). Nella verità cristiana si dissolvono questi contrasti apparenti per comporsi in quella lieta gravità, sgombra d'ogni tragicità, per la quale Clemente d'Alessandria ha definito la vita „divin gioco da fanciulli” (ibid., p. 14). L'uomo cristiano, il homo ludens supera ogni tragicità verso una sublime regione teologica dove le parole e i rapporti debbono essere sapientemente ponderati per evitare l'impressione di voler defraudare della sua abissale serietà il mondo della verità cristiana. I Padri e i teologi parlano della „Chiesa che gioca" dunque dello spazio fisico-spirituale nel quale il Logos fatto uomo esegue il suo „gioco della grazia”, e dove il cattolico che non si è ancora perduto in un invadente intellettualismo conciliando mirabilmente la trasfigurazione operata dalla grazia col suo permanere uomo risponde al gioco della grazia nel gioco dei suoi sacramenti e della sua liturgia. Sicché Chiesa e grazia e azione liturgica gli 
sono pre - „ludio” di quella libera serenità che avrà la sua evidenza nell'eterno gioco della visione divina.

\subsubsection{Deus ludens}

L'homo ludens può essere compreso solo se anzitutto, e con tutto il timore reverenziale possibile si parla di un Deus ludens (ibid., Capitolo I). Quando si dice che Dio creatore „gioca”, si esprime metaforicamente una visione metafisica per cui la creazione del mondo e dell'uomo sono un atto divinamente logico ma in nessun modo necessario a Dio. Delimitando con la definizione „logico ma non necessario" l'intima essenza del gioco divino, l'atto creativo di Dio non si priva in alcun modo della sua immensa gravità (consistente appunto nell'insondabilità del significato). Il Creatore è libero e dall'infinita pienezza dei possibili ordini Egli ne suscita all'esistenza uno determinato genialmente eletto (se è lecito parlar così di Dio) e formato perché in esso e per esso siano visibili la sua sapienza e la sua bontà. La creazione gioca innanzi a Dio il suo gioco cosmico dal moto degli atomi e delle stelle fino al grave e bel gioco del genio umano e fino alla danza beata in cui si inseriscono gli spiriti che tornano a Dio.

La massima profondità dell'uomo ludico consiste nel fatto che ha scrutato i veri rapporti di tutte le cose terrene volgendo l'occhio a Dio,l'essenza di tutti gli esseri, che valuta l'uomo e la sua sorte non più e non meno gravemente di quanto si addica ad un meraviglioso giocattolo. A chi dunque considera Dio con gravità non è lecito considerare altrettanto gravemente la creatura umana come se in essa si concludesse tutto il significato delle cose: e chi avrà compreso la trascendenza insita nell'essenza dell'essere creaturalmente partecipante, potrà „giocare" poiché ormai conosce il giusto mezzo tra serietà e scherzo, fra tragicità dell'esistenza e sereno fiducioso abbandono al gioco della vita, diretto dalla bontà di una sapienza ludica. Le varie cose del mondo e tutto quanto accade sono trasparenti se riferiti al Dio che gioca; a Colui che è, anche infantilmente, onnipotente e onnisciente, Dio appunto, che „giocando" tutto suscita.

Il „gioco di Dio” si rende accessibile alla creatura mediante la creatrice alienazione di se stesso nel meraviglioso gioco delle sue opere che ha plasmato con le variopinte immagini del suo mondo un „gioco da bambini” per educare l'umanità alla „gravità” dell'eterno e dell'invisibile. Tutto il gioco che il Logos realizza sul globo per l'estasi del Padre, la sua danza cosmica sul mondo, non è che un'allusione giocosa a ciò che dai primordi era nei divini archetipi dell'eterna sapienza e che si svelerà quando la danza della terra finirà (cfr. ibid., p. 28).

\subsubsection{Homo ludens}

L'homo vere ludens non può essere altro che un uomo dalla serietà dell'aldilà, non può essere né un corrotto dal gioco né un disperato. L'uomo che ha colto la trascendenza di Dio di fronte a tutto il creato non prende sul serio, falsandole, neanche le cose più serie. Non solo la serietà deve saper giungere alla radice delle cose, bensì ancor più facilmente vi possono giungere scavando più a fondo appunto per gioco la serenità, l'ironia e l'umorismo (ibid., Capitolo II).

Questa seria serenità fiorisce solo nell'equilibrio tra cielo e terra: in un uomo che ama il mondo multiforme e pur ne sorride, che ne conosce l'origine da Dio e anche i limiti. Solo chi ha spiritualmente conciliato questi opposti è in verità un homo ludens. Se egli fosse solo ludens, lo dovremmo definire un giocatore perdutosi per leggerezza; se fosse solo homo sarebbe un disperato. L'homo ludens è sintesi, colui che da,,serio e sereno" e da umorista disinvolto sa sorridere anche tra le lacrime e trova in fondo a tutta la serenità terrena la faccia dell'insoddisfazione. L'esistenza è lieta (perché raccolta in Dio) e tragica (perché pericolosamente libera). Essa è del pari gioia e sofferenza, commedia e tragedia. L'homo ludens è ,serio-sereno”, è quello che i greci con un termine quasi intraducibile hanno definito anér spoudogeloios (cfr. ibid., p. 32). Questo uomo è sempre duplice: un 
uomo dal sereno distacco spirituale, un uomo per così dire dall'anima elegante, invincibilmente protetto; e tuttavia un uomo tragico, che ride e piange. L'homo ludens è perciò un uomo serio perché conosce contemporaneamente tanto il significato quanto la non-necessarietà della sua esistenza reale.

Saprà centrare questo equilibrato gesto di giocatore di razza con sicuro istinto solo quell'uomo che conosce l'esatto punto a mezza via tra terra e cielo: dunque non chi disprezza cinicamente la terra o la desidera epicureamente, bensì chi è anche tanto sollecito del divino da saperlo trovare proprio in mezzo alle cose terrene. In altri termini la vera serenità di un uomo che gioca e per cui la serietà e lo scherzo sono fratelli è un problema religioso e può essere vissuto solo da un uomo terrestre-celeste (cfr. ibid., p. 37). Sarebbe proprio questa l'arte sovrumana di una cultura umana dell'anima: saper ancora giocare con serietà e in ogni gioco non dimenticare la serietà. Darsi alle cose senza disprezzarle in un'evasione dal mondo... e tuttavia esser liberi e distaccati perché esse non debbono essere prese troppo sul serio. L'uomo invece al centro tra cielo e terra, il vero gnostico, il cristiano, può cogliere tutta la sua vita e ogni accadimento nel mondo come un unico grande teatro (poiché conosce i segreti che guidano la scena), senza perciò precipitare nel dolore o nell'ansia del mondo: serietà e gioco gli sono fratelli in Dio.

„Così lo gnostico, nello spettacolo della vita, recita inappuntabilmente la parte che Dio gli ha assegnato, perché sa cosa fare e cosa sopportare,,

(ibid., p. 41)

- dice Clemente Alessandrino. Solo all'uomo radicato nella realtà di Dio è dunque concesso dire che questa vita terrena altro non è che gioco e ombra. Poiché solo a lui, che crede che questo mondo scaturisce dalla pienezza dell'Essere divino, è dato esprimere immediatamente col sì anche il no, è dato gettare il mondo poco fa accettato con amore e abbracciato (compreso se stesso) perché è opera di Dio come un infantile gioco ozioso per slanciarsi verso la „serietà-beata,, che esiste solo in Dio.

„Ci pare infatti che solo per la fede nella vera incarnazione di Dio ci sia data in assoluto la possibilità di conquistare quella 'serena sicurezza e libertà' senza cui (come si è già detto) non è possibile un gioco libero"

(ibid., 38)

- aggiunge H. Rahner.

\subsubsection{Ecclesia ludens}

Dove Dio irrompe direttamente come uomo nel nostro sistema di gioco, apparentemente tanto chiuso, tutte le nostre parole cedono. Non ci resta che parlare con Paolo della „follia” e della „debolezza” di Dio, oppure col grande maestro Gregorio del „gioco” di Dio, ben sapendo che anche questi tentativi non giungono nei pressi della verità totale neanche se parliamo della „sapienza”, della „forza” e della „serietà” di Dio (cfr. Capitolo III). Il Dio del creato, la cui opera si osò definire un „gioco" perché pienamente significante e non necessario, si è poi dato anzi consegnato alla propria creatura umana in un modo che travalica essenzialmente ogni creazione: nell'incarnazione e con ciò nell'opera sgorgata dall'amore più libero, che si definisce chiesa, grazia, visione di Dio. E poiché si tratta di un dono scaturito dalla divina genialità dell'amore, dono che lo spirito creato, anche se nell'ordine della grazia, non saprà mai valutare, dono che non è dovuto ad alcuno e al quale l'uomo in quanto figlio di Dio risponde con amore altrettanto libero e invalutabile. Con i sublimi spiriti del misticismo è lecito definire ,gioco" anche questo accadimento umano e divino, anzi con una motivazione ancor più profonda che per l'opera della creazione perché nel gioco della grazia Dio è divenuto in Cristo addirittura partecipe del gioco. E quando questo gioco della grazia volge al termine, quando tutto (fede, speranza, legge, sacramento) cessa, allora ha inizio il divin gioco infantile della visione eterna - come diceva Clemente -, la grande panegyris, l'assemblea plenaria con Dio, la festa in cielo (cfr. ibid., p. 47). 
Fede e gioia di redenzione del cristiano battezzato, dell'eterno bambino generato da Dio sono dunque un autentico gioco infantile e la chiesa acquista bellezza dai passi di danza il cui ritmo è segnato dal sacro Pneuma. Nella sua profondità poetica e teologica questa visione della chiesa che gioca e che simboleggia l'unione di tutti coloro che sono diventati felici in Cristo insegna a spingere lo sguardo al di là delle cose visibili: sole, luna, acqua, olio e vino, al di là della storia apparente, verso ciò che Dio ha inteso, verso l'eterno. Ella è un gioco-sacramento. Questa chiesa incessantemente attingendo alla fede nell' Incarnazione salva l'unità tra spirito e corpo, idea e simbolo, invisibile e visibile e proprio perciò considera ogni cosa visibile come un incessante gioco di Dio, come un "atteggiamento del corpo" in cui si esprime senza sosta la gioia dello Spirito Santo (cfr. ibid., p. 52).

Chiesa e grazia infatti sono solo un preludio terreno di quella fine che è l'eterno principio della beatifica visione di Dio. Pre-,,ludio": infatti tutto l'aldiqua non è che un gioco infantile, per quanto serio e decisivo, confrontato all'armonia eternamente sicura dell'anima e del corpo che per noi è cielo. Il gioco del cielo sarà un gioco dello Spirito Santo, un eterno librarsi col Pneuma che „spira dove vuole".

\subsubsection{Il gioco celeste}

Il gioco autentico e la letizia senza fine hanno inizio soltanto al di là della terra (ibid., Capitolo IV). In fondo di ogni gioco c'è un mistero e tutto, dal gioco infantile al gioco celeste, accenna a quella beata serietà di cui è degno solo Dio. Il bambino che gioca è sempre un insopprimibile duplicità di debolezza e forza, di serietà e scherzo. Desidera più d'ogni altra cosa la libertà, la pace, la disponibilità dello spirito e la serenità dell'anima; si attende un gioco, un essere infantile al sicuro, inoltre quella levità che slancia il corpo liberato dalla gravità terrena verso una danza celeste. Nel gioco la bella e serena levità dello spirito cerca l'espressione per la quale l'uomo aspira alla spiritualizzazione e all'entusiasmo. Il gioco nella sua intima radice e nei suoi frutti è un mistero sacrale, la speranza in un'altra vita che diventa un gesto e un atteggiamento. Il gioco è incantesimo, rappresentazione del totalmente altro, anticipazione del futuro, rifiuto dell'oggettività opprimente. Quanto è terreno nel gioco diventa improvvisamente provvisorio, presto superato e infine del tutto eliminato: e lo spirito è così pronto ad accettare l'inaudito, ad addentrarsi in un mondo di leggi del tutto diverse, pronto ad essere senza peso e libero, regalmente indipendente e divino. L'homo ludens attende, come si è già detto, quella miracolosa levità che spinge alla danza celeste il corpo liberato dal peso terreno. Ogni gioco in qualche recesso della sua essenza è una danza, un girotondo attorno alla verità (cfr. ibid., p. 63). Il gioco sacrale fu sempre danza. La danza è sacra se è gioco, poiché è anzitutto imitazione nel gesto e nel ritmo di quello slancio che Dio ha ispirato al cosmo quale principio creatore.

Secondo la nostra teologia del gioco celeste l'uomo redento è ridiventato bambino come lo era all'istante della sua nascita terrena e della generazione nel primo sacramento della chiesa, perciò egli gioca.

\subsection{La discussione e la storia degli effetti dei libri di Huizinga e Rahner}

L'opera di Huizinga ebbe ed ha ancora un grande eco. Vedendo la storia dei suoi effetti il Homo ludens può essere considerato un'opera di base delle teorie sul gioco che è stato commentato, sia positivamente sia negativamente, da tutti i teorici del gioco. ${ }^{13}$

In breve la filosofia e la concezione teoretico-culturale del gioco nell'opera Homo ludens può essere valutata come una „ontologia culturale" notevole il cui autore cercava di fondarla sulle basi umanistiche. Questa „ontologia culturale” nella sua elaborazione filosofica è un poco grossolana ed è

\footnotetext{
${ }^{13}$ Gli autori più importanti dell'elaborazione della filosofia del gioco sono: CAILLOIS 1967; 1981; FINK 1957; 1969 \& 1960; 1969b; GADAMER 1960; 1983.
} 
concentrata esclusivamente sull'uomo nella sua visione sul gioco. Riguardo al rapporto cultura-gioco Huizinga prende una posizione estrema e non può difenderla dall'accusa di „panludismo” ed esiste un'altra insufficienza della sua teoria in quanto non può fornire nessuna spiegazione sull'origine del carattere trascendentale del gioco. Evidentemente questa intuizione ha spinto Hugo Rahner a scrivere un'opera complementare ed omonima con quella umanista di Huizinga. Hugo Rahner nel suo libro dà un esempio brillante della complementarità di teologia ed antropologia quando non soltanto continua ad analizzare i temi riguardanti i riferimenti religiosi tirati in ballo da Johann Huizinga ma li rende l'asse teologico dell'intera concezione homo ludens ed argomenta fortemente a favore della ragione d'essere di questi temi da egli analizzati. ${ }^{14}$

Jürgen Moltmann (nato nel 1928), docente di teologia sistematica alla facoltà evangelica di teologia all'università di Tubinga dal 1967, scrisse il suo libro intitolato Sul gioco negli anni della contestazione studentesca (1968/69).

\section{„L'ho scritto semplicemente perché mi andava di farlo [...]. Ciò che in questo libriccino è importante non è quel che è necessario ma soltanto ciò che ̀̀ bello.” (MOLTMANN 1988, p. 109)}

Il libro di Moltmann tratta una „teoria critica del gioco" per anticipare - giocando e sperimentando quella che dovrebbe essere una vita liberata in mezzo a questa vita di coercizioni e doveri. „Perché Dio ha creato il mondo?" - si chiede il bambino che non è più tale. Egli sa che tutto nel mondo degli adulti ha le sue buone ragioni ma anche il mondo ha una ragione? L'interrogazione metafisica si è qui imbattuta fin dal principio nell'aperto, nello sconfinato o nell'abissale. A partire da Eraclito il gioco è stato spesso usato come simbolo cosmico del mondo. La fede risponde infantilmente alla non infantile domanda del fanciullo e la sapienza della teologia termina con la libertà dei figli di Dio. Siamo così già entrati nel gioco liberatore della fede, con Dio contro la cattiva pressione della paura e il grigio stimolo della preoccupazione che la morte ci mette addosso. Infatti la fede nella resurrezione è il coraggio di resistere all', alleanza con la morte” (Is 28,15), è speranza nella vittoria della vita che inghiotte la morte, contro la morte che divora la vita. La croce di Cristo non appartiene al gioco bensì rende possibile il nuovo gioco della libertà. La fede è una nuova spontaneità e una spontaneità più sveglia. Per i peccatori il redentore è necessario, egli però viene spontaneamente e pertanto porta oltre la miseria e la sua redenzione, verso un futuro libero che egli stesso dona. La nuova creazione, che promana dalla redenzione non è l'antica creazione ristabilita o puramente riparata ma è qualcosa di nuovo anche rispetto alla prima creazione. Essa è un nuovo gioco. Di fronte alla croce di Cristo „è tutto inutile”, dice il nichilista e dubita. Nella stessa situazione il credente dice ,è veramente tutto gratuito", e si rallegra della grazia che gli dà tutto gratuitamente e spera in un nuovo mondo in cui tutto si dà e si ha gratuitamente.

"Quando l'uomo si sente libero e desidera usare la sua libertà, la sua attività diventa un gioco", dice Sartre al seguito di Schiller (MolTMANN 1988, p. 38). Partendo da questo gioco può essere chiarita la differenza tra l'homo faber e l'homo religiosus. Il mondo come storia è il simbolo necessario dell' homo faber. Il gioco come simbolo cosmico accoglie certamente delle rappresentazioni arcaiche ma supera il mondo come storia se in tal modo si risale non solo alle origini del pensiero ma anche all'escatologia dell'essere. Il gioco è il simbolo dell'homo religiosus (ibid., p. 94) perché come simbolo cosmico trascende le categorie del fare, dell'avere e delle prestazioni per raggiungere quelle dell'essere, dell'autentica esistenza umana e della gioia dimostrata in essa. In altre parole, di fronte al produttivo si afferma il creativo e di fronte all'etico si afferma l'estetico. Il pesante lavoro terrestre viene trasformato in gioia, danza, canto e gioco e ciò fa molto bene anche al lavoro.

\footnotetext{
${ }^{14}$ Tra gli autori importanti che hanno contribuito all'elaborazione della teologia del gioco ricordiamo: BALTHASAR 1983; 1978; COX 1969; 1971; GANOCZY 1992; 1997; HOUGH 1997; MolTMANN 1971; 1988 \& $1985 ; 1986$.
} 
Harvey Cox (nato nel 1929), anche lui teologo protestante, dal 1966 insegna alla Harvard Divinity School. Tra le sue pubblicazioni è molto noto il saggio su La festa dei folli (1968) il cui tema è la libertà ludica come libertà profetica che non cerca accomodamenti o banalizzazioni, confusioni omologanti o appiattimenti, ma una dimensione del totalmente nuovo e quindi anche crisi e rottura. In questo senso H. Cox interpreta la figura del Cristo nell'ultimo capitolo del suo controverso libro come Cristo arlecchino.

Come è noto, mediante la categoria ludica del clown vuole focalizzare il carattere trasgressivo del Cristo, l'unico a suo parere che può ancora parlare all'uomo di oggi divenuto sordo alle parole del Cristo maestro di saggezza, indifferente al Cristo giudice, incredulo di fronte al Cristo salvatore.

In un mondo che sembra aver rimosso le categorie della fantasia e dell'immaginazione, della festa e del ludico, in quanto perso dietro il criterio dell'utile, il Cristo può essere la personificazione di tutti questi sensi perduti ma l'identificazione con il clown va colta con un ulteriore approfondimento, anche oltre il testo di Cox. Si deve accettare l'immagine da lui proposta per scoprire cosa si nasconde sotto di essa e sotto la maschera del clown. Consapevolezza della maschera e denuncia di essa e tentativo di strapparla: questa è anche l'ambiguità del gioco che si muove tra apparenza velata e realtà svelata, tra presenza ed assenza, tra „trasparenza ed ostacolo”. Cox infatti sottolinea solo l'aspetto di trasgressione del Cristo arlecchino, cioè del Cristo che sfida le consuetudini e disprezza il potere, satireggia sull'autorità costituita facendone addirittura l'imitazione nell'ingresso a Gerusalemme, a cui corrisponderà un'estrema derisione, il capovolgimento finale ad opera dei suoi nemici che gli imporranno le insegne reali e lo crocifiggeranno tra risate ed insulti. Qui si esprime tutta l'ambigua complessità del clown, il versante tragico ineliminabile da questa figura. Da un lato il clown o l'artista è colui che ha fatto della propria vita una recita e tale identificazione è già un passaggio dal mondo quotidiano, dall' apparenza ordinaria, ad una nuova apparenza, al mondo fittizio del circo (o del gioco) violentemente insensato ma allettante, una macchia colorata nel grigio di una società o di una mente, la cifra di una mitologia alternativa, espressione di un'anima vagante o giocoliere pasticcione e sfortunato. Ma d'altro lato il clown (e l'artista) con il mancato senso del suo gioco assume il valore di una messa in questione, di una sfida a certezze codificate; con l'affermazione di una contraddizione e con la presenza di un vuoto è „vocazione dello slancio e della caduta, dell'altitudine e dell'abisso, della Bellezza e della Sventura" secondo le parole di Baudelaire.

Il Cristo arlecchino allora lascia trasparire (pur nascondendo) un'angoscia disperata e incarnando il ruolo di vittima, anche grottesca, manifesta una fragilità estrema, ossia la vertigine mortale in cui si trova esposto, una sorta di mancanza d'essere che se per il clown è legata alla natura illusoria dell'arte, per il Cristo rinvia all'accettazione della condizione umana.

Negli ultimi due decenni in corso del dialogo interdisciplinare tra la teologia e le scienze naturali è stata delineata la possibilità di una teologia della natura in base alla simbologia del gioco. Tra i teologi autori più notevoli del tema (Alexandre Ganoczy, Michael Hough, Jürgen Moltmann e Gisbert Greshake), quest'ultimo nella trattazione relativa al malum morale si richiama anche nel caso del malum physicum alla libertà concessa da Dio alla creazione per spiegare teologicamente l'origine della sofferenza:

"Un progetto preliminare di strutture di libertà esiste già nel mondo evolutivo preumano, e precisamente lì ove esso non appare come definito e determinato, bensì si sviluppa per tentativi nel libero gioco delle forze, lì ove il causale interrompe continuamente il necessario. Queste riflessioni offrono una base per comprendere il fenomeno della sofferenza, della disintegrazione, del mancato sviluppo, della durezza del mondo. Il fatto che esistano cose come il tumore, malattie virali, esseri deformi, aborti, incidenti, inondazioni e simili è una conseguenza necessaria di una evoluzione che procede sotto forma di progetto 
preliminare della libertà, non deterministicamente, non necessariamente, non fissamente, bensì attraverso il gioco e la sperimentazione di possibilità, in modo casuale." (GRESHAKE 1988, p. 43)

Questo gioco del mondo creato comprende il ruolo creativo del „caso”, lo capisce cioè come evento del nuovo indeducibile, quello verso cui il vivente si rapporta in modo ludico e che accetta per il libero gioco delle forze disponibili. Il „,caso” non sarà allora qualcosa di irritante perché sfugge alle nostre possibilità di calcolo. Anzi, la necessità delle leggi costituirà soltanto un reticolo aperto di atteggiamenti modellati secondo le esperienze del passato, quelle che ci consentono di percepire ed accogliere le possibilità che il futuro ci riserva. Le forme del gioco per edificare dei sistemi vitali più complessi comprendono entrambi: necessità e caso, legalità e libertà, realtà e possibilità, passato e futuro.

Con la realizzazione della trilogia composta dall'estetica, dalla drammatica e dalla logica teologica Hans Urs von Balthasar (nato nel 1905) ha saputo ricostruire quell'anello mancante della tradizione teologica che poteva leggere l'evento della rivelazione alla luce dei tre trascendentali. La simbologia della sua Teodrammatica (1973-1983), rende possibile di tentare un'interpretazione ludica della storia della salvezza. Dio conduce il suo gioco sul palcoscenico del mondo, il gioco del suo amore inesauribile e inventivo e nella storia del mondo egli conduce il gioco della grazia che deve rivelarsi in assoluta libertà a tutte le creature. Se Dio fondamentalmente si rappresenta sulla scena del mondo, il suo gioco sarà allora un'autorivelazione. In tutte le creature e attraverso tutti i ruoli da esse svolti ciò che Egli intende è appunto rappresentare se stesso e gli esseri umani sono le sue immagini, il suo riflesso, i suoi ruoli e maschere, le sue ,persone” in questo grande teatro del mondo. Il disvelamento della sua realtà nascosta con l'autorivelazione è il suo manifestarsi in forma umana. Ora viene disvelato il senso nascosto dell'intero come anche il senso celato in qualsiasi ruolo svolto dagli uomini. Ora si possono cogliere pure il fine e la fine del grande teatro del mondo: quando apparirà il regno della gloria, anche la natura verrà condotta alla libertà della vita eterna.

La teologia e la filosofia del gioco formulano un proprio linguaggio filosofico-teologico che è un mezzo utile per un'analisi contemporanea di alcuni enunciati della fede. In quanto è compito della teologia fondamentale di condurre una ricerca sulle possibilità dell'espressione del depositum fidei in un modo opportuno ed adeguato per l'uomo di oggi, il confronto con J. Huizinga e H. Rahner è un confronto di carattere teologico fondamentale in quanto permette di elaborare un discorso teologico con strumenti concettuali più attuali e rispondenti alla nostra epoca.

\section{La sfida postmoderna: verso una teologia ludica...}

La possibilità di una teologia ludica può essere esaminata partendo da alcuni brevi cenni sulla filosofia di Nietzsche tramite il riassunto della tradizione connessa col gioco e tramite le conclusioni da esso tratte.

\subsection{Il nichilismo ludico di Nietzsche}

La trattazione della simbolica del gioco e dell'elaborazione teologica di essa le quali intendono delineare la comprensione teologica dell'homo ludens sulla scia di Hugo Rahner si basano sull'antropologia ludica di Johann Huizinga. La simbolica del gioco però non è congelata alle manifestazioni dello scatenamento infantile bensì le si associa la "corse in folle" di una vita diventata insensata. Quest'ultima ambiguità rende la filosofia del gioco un argomento serio ed attuale nella nostra epoca postmoderna. Il gioco può diventare il simbolo veramente autentico del mondo soltanto in tale contesto paradossale. L'ermeneutica della simbolica del gioco entrata nella conoscenza comune 
con Nietzsche e con i suoi commentatori, ossia l' interpretazione cinica e nichilistica offre un punto di vista opposto all'interpretazione teologica qui delineata e si presenta come una vera sfida apologetica.

Secondo un suggerimento di Heidegger si deve „pensare l'essere e il fondamento a partire dal gioco e non il gioco a partire dalla ratio" (BREZZI 1992, p. 4). Dal frammento 52 di Eraclito ad Heidegger, commentatore di quel passo, il gioco è stato presente con alterne vicende sulla scena filosofica, ha giocato la sua partita combattendo tuttavia sempre una battaglia contro la pretesa di una ragione logica e sistematica che tentava di emarginarlo e con esso di annullare tutte quelle tematiche connesse: il riso, il comico, il sogno, accusate di insignificanza cognitiva. ${ }^{15}$

Secondo Fink il gioco non è un tema fra tanti ma argomento fondamentale ed originario come la morte, l'amore, il lavoro e la lotta; fondamentale in quanto è rapporto di senso tale da aprire una dimensione altrimenti preclusa. ${ }^{16}$ Esso si schiude infatti all'altro, sia esso Dio o il mondo (1969b, p. 287; ed anche 173ss.).

Fink scrive di Nietzsche:

"Un'origine non metafisica di filosofia cosmologica si trova nel suo pensiero del 'gioco'; già partendo dai suoi primi scritti egli si muove nella misteriosa dimensione del gioco, nella sua metafisica dell'Artista, nella sua immagine eraclitea di Zeus, figlio del mondo, che gioca, del pais paizon." (FINK 1993, p. 203)

Per Nietzsche il Gioco umano, il Gioco del bambino e dell'artista diviene il concetto chiave dell'universo e una metafora cosmica. L'uomo ha possibilità di comprendere l'apparenza come apparenza, di immergersi a partire dal suo proprio gioco nel grande gioco del mondo e di sapersi compagno di gioco del gioco cosmico in tale sprofondare. Quando il gioco del mondo è al centro dell'attenzione e quando l'intuizione scopre l'inganno ,apollineo”, l'uomo intuisce le creazioni dell'apparenza finita e giunge a vedere la „vita” che crea; quando costruisce, distrugge; quando il sorgere e il decadere delle opere limitate e temporanee vengono sperimentate come danza e girotondo, come gioco di dadi dei capricci divini sotto la volta del Paradiso, dell'innocenza e della mancanza di pericolo - allora l'uomo, nella sua produttività giocosa, può sentirsi legato alla vita del tutto, ammesso nel grande gioco di nascita e morte di tutte le cose e coinvolto nella tragedia e nella commedia dell'esistenza universale. Nell'Ecce homo Nietzsche dice:

„Non conosco altra maniera di trattare i grandi compiti che non sia il gioco: fra i segni della grandezza, questo è un presupposto essenziale."

(Ibid., p. 205)

L'uomo che gioca, che è estaticamente aperto „al dio Dioniso", che non ha forma e che crea forme e che gioca, non vive nel vago arbitrio di un'assoluta libertà; egli è compagno di gioco del gioco del mondo e vuole fin nell'intimo il Necessario. Per tale volontà, che non è abbandono ad un destino ma partecipazione al gioco cosmico, Nietzsche usa la formula amor fati.

\footnotetext{
${ }^{15}$ Tra i filosofi antichi e moderni che hanno trattato questo tema i più illustri sono: Eraclito, Kant, Schiller, Hegel, Nietzsche, Heidegger e ai nostri giorni, Fink, Gadamer e Axelos.

${ }^{16}$ Eugen Fink (1905-1976) ha insegnato per molti anni Filosofia a Friburgo, ricoprendo la cattedra che era stata di Husserl e di Heidegger. Considerato uno degli esponenti più significativi della scuola fenomenologica husserliana è stato successivamente influenzato dal pensiero di Heidegger assieme al quale nel 1970 ha pubblicato il famoso seminario su Eraclito. Tra i suoi libri più importanti per noi: La filosofia di Nietzsche (1960; 1993) e Il gioco come simbolo del mondo (1960; 1969b).
} 


\subsection{Il gioco sacro del popolo di Dio}

Ciò che accade nel battesimo, nella misteriosa nascita per mezzo dell'acqua e dello spirito - dice Beda Venerabile - è un mistero noto solo alla fede, dall'esterno non è che immagine e gioco. Sola haec Ecclesia Mater, quae generat, novit. Caeterum oculis inspicientium videtur exire de fonte qualis intraverit, totumque ludus esse videtur, quod agitur.

„Solo la madre chiesa che genera, sa cosa accade. Agli occhi dello spettatore colui che si calò nel fonte battesimale ne esce come vi era entrato e tutta l'azione non sembra essere altro che un gioco."

(RAHNER 1969, p. 53)

Qui si cela la radice teologica dell'insegnamento di Romano Guardini circa la liturgia come gioco divino.

\subsubsection{Romano Guardini sullo spirito della liturgia}

Romano Guardini è una delle figure più significative e più profonde fra i pensatori cattolici del Novecento. La sua opera esprime una grande consapevolezza del proprio tempo, che è passato con forza eccezionale attraverso il suo spirito con il senso dei suoi problemi più veri e più seri. $\mathrm{Al}$ centro di tutta l'opera guardiniana c'è l'analisi dell'eredità dello spirito moderno e il duro confronto con la pretesa di autonomia sulla quale si fonda la modernità. ${ }^{17}$

Guardini ha assunto un importante ruolo nella storia cristiana dello spirito del XX secolo. È uno dei più significativi pionieri che hanno tratto fuori la Chiesa cattolica in Germania dal ghetto intellettuale $\mathrm{e}$ culturale introducendola in una nuova epoca. L'opera guardiniana ha avuto un grande impatto sulla cultura, non solo in Europa, ma anche in America, dove ha influenzato notevolmente i più grandi intellettuali cattolici (Flannery O'Connor, Dorothy Day, Thomas Merton).

Le intuizioni del famoso libro di Guardini intitolato Lo spirito della liturgia (1918) non solo prepararono il terreno alla svolta del concilio Vaticano II ma gli consentirono la nomina nel 1961 a membro della Commissione liturgica preparatoria del Vaticano II. Romano Guardini spiega nel suo libro che la liturgia è „gioco" cioè uno spazio slegato da qualsiasi utilità pratica. Proprio perché la liturgia è „gioco” essa rinvia al primato di Dio, alla priorità dell' ascolto-visione che pone l'uomo nella logica dell'affidarsi.

\subsubsection{Il gioco liturgico}

La liturgia è un „gioco drammatico" ma non uno spettacolo per spettatori estranei, bensì un evento drammatico che coinvolge e trasforma i singoli spettatori. Nella celebrazione del sacramento simbolo e parola sono tra loro dinamicamente uniti in un modo che permette di parlare di gioco drammatico che trasforma i partecipanti. Il gioco (Spiel) è un attività non utilitaristica ma proprio in questo modo

\footnotetext{
${ }^{17}$ Guardini nacque a Verona il 17 febbraio del 1885. L'anno successivo la famiglia si trasferì a Magonza, in Germania, dove il padre seguì i suoi affari commerciali, svolgendovi pure le mansioni di Console per il Governo italiano. Conseguita la maturità classica, Romano, cercando di trovare la propria strada, studiò Chimica a Tubinga e, successivamente, Scienze Politiche a Monaco di Baviera e a Berlino. Dopo due anni, ebbe luogo la sua crisi religiosa $o$, più profondamente, l'irruzione di una religiosità più forte e più personale. La riscoperta della fede coincise con la sua vocazione sacerdotale. Nel 1906 si iscrisse alla Facoltà teologica di Friburgo e poi in quella di Tubinga. Dopo due anni entrò nel Seminario teologico della sua diocesi di Magonza e nel 1910 venne ordinato sacerdote. Dopo due anni di attività pastorale, Guardini riprese i suoi studi teologici e dopo tre anni conseguì il dottorato in teologia all'Università di Friburgo. Dal 1923 fino al 1939 insegnò nella capitale tedesca. Nel novembre 1945, dopo gli anni del silenzio, Guardini fu reintegrato nella sua cattedra, non più a Berlino ma alla Facoltà filosofica dell'Università di Tubinga, dove insegnò per tre anni. Nel 1948 accettò la chiamata dell'Università di Monaco di Baviera e lì tenne la cattedra fino al 1962. Morì il 1 ottobre 1968.
} 
efficace. Il „gioco" non è l'opposto della „serietà”, ma del modo di agire utilitaristico (cfr. SCHNEIDER 1995b, p. 252)!

Il senso della liturgia, senso ad essa interiore, è la sublime inutilità che emerge con chiarezza non appena ci si avvicini ad essa con comprensione particolare e senza pretese di spiegazioni esaustive (GUARDINI 1919; 1987). La liturgia pertanto che non va considerata semplicemente come una tappa del cammino spirituale, anche se si può cogliere nei riti e negli atti liturgici un valore formativo (ma come afferma Guardini, non come metodo educativo „progressivo e calcolato”), crea un' atmosfera spirituale in cui l'animo cresce e sviluppa. La liturgia infatti è un mondo di vita concluso in se stesso con un suo spazio e un suo tempo particolare, come il gioco, nel quale si realizza il rapporto uomoDio e si ripete, rammemorando, l'Alleanza.

Lungi dall'attribuire alla liturgia un significato morale o moralistico la si deve vivere come luogo di esistenza religiosa, il centro in cui ascoltando e pregando si celebra la Parola di Dio. Una liturgia quindi come comunione tra Dio e uomo sotto il segno della gratuità e della gioia, del godimento sereno, che si ispira al detto di Giovanni „Lui per primo ci ha amati” (1Gv 4,10): da questo primo dono nasce la preghiera e la possibilità del dialogo dell'uomo con Dio. Il punto di partenza non è pertanto un generico bisogno religioso, né una vaga elevatio mentis in Deum, di tipo platonico o plotiniano (seppure sfumature di tale aspetto talvolta si ritrovino) ma l'amore di Dio per l'uomo, una sorta di autocomunicazione dell'Infinito, a cui la preghiera segue come risposta ad una chiamata e diventa a sua volta atto d'amore e assume in sé anche il naturale bisogno religioso dell'uomo.

Di conseguenza, definire la preghiera e tutta la liturgia come ascensione verso una comunione con Dio sta a significare un preciso riferimento all'Alleanza, al Dio e all'uomo che in quell'atto si sono incontrati e ludicamente si incontrano ancora.

Tutti i temi classici dell'antropologia biblica riassumibili nella theopoiesis dell'uomo si ritrovano in questa specie di cooperazione ludica che è la liturgia: in essa è presente quel misterioso legame tra Dio e uomo che fa sì che questi vive in una realtà di segni e gesti divini, di verità sacre che non possono essere demitizzate ma vissute come trasposizione sulla terra della vita dei cieli, di quella vita di cui parla la Sapienza nei Proverbi o Ezechiele nella sua visione (cfr. GUARDINI 1987, pp. 213-214).

Similmente all'arte (e alla vita infantile), la liturgia realizza la fragile unione di sogno e realtà, l'intreccio di desiderio e possesso, di essere e dover essere, di corpo e di spirito:

\section{„Sublime unione di profonda serietà e divina allegria... non lavoro, ma gioco, $e$ si tratta di condurre il proprio gioco davanti a Dio, non solo creare, ma essere essa stessa un'opera d'arte." (Ibid., p. 220)}

L'uomo che conduce il proprio gioco davanti a Dio, è colui che ha rinunciato alla prudenza dell'adulto, alla razionale utilità, all'esasperato funzionalismo e al contrario non ha vergogna, come David davanti all'arca, di danzare e giocare, consapevole anche dello scherno da parte dei saggi: l'uomo dunque che seguendo la parola del Cristo diventa bambino e come tale porta avanti il suo gioco. D'altra parte la liturgia manifesta anche che il Dio che partecipa a tale ludus, come abbiamo già detto, assume connotazioni nuove ed evita il pericolo di una eccessiva dilatazione dei suoi spazi, non presentandosi come pienezza di essere.

Tutta la preghiera e la liturgia, possono essere considerate in prospettiva ludica il primo effetto della grazia e allo stesso tempo il raggiungimento di quella come scopo.

\subsection{Il gioco nella Tradizione cristiana}

Il Signore glorificato viene a noi non soltanto nella celebrazione eucaristica, nel ,gioco" augusto della liturgia sacra, ma anche nella sua parola, nel prossimo, nel momento presente col suo ruolo ben definito, nei piccoli e più insignificanti avvenimenti di ogni giorno. Il Cristo glorificato vuole allenare 
i discepoli e noi tutti al gioco divino che desidera giocare con i redenti; vuole iniziarci alla vita di fede, farci comprendere che dobbiamo cercarlo: con dolore, come Maria e Giuseppe hanno cercato il Bambino Gesù smarrito (Lc 2,48), con lacrime, come Maria Maddalena (Gv 20,11.13.15). Noi sappiamo che è presente, vicinissimo a ciascuno di noi:

„Ecco, io sarò con voi tutti i giorni, sino alla fine del mondo.”

Il Cristo glorificato realizza ciò che in apparenza è contraddittorio: va al Padre - e viene a noi (Gv $14,28 ; 14,3.18)$; se è nascosto ai nostri occhi corporali, ci è però vicino, visibile agli occhi della fede (Ef 3,17); si mostra sotto i travestimenti più diversi dietro i quali la nostra fede è invitata a riconoscerlo. Dio si nasconde da noi ma ci cerca anche nello stesso tempo. Lui ama il gioco perciò l'idea del „gioco” è presente implicitamente in tutta la Bibbia (LAFFEY 1999, pp. 187-195). È un mistero sconvolgente che il nostro Dio sia vicino a noi, più intimo di noi stessi, e tuttavia completamente nascosto. Sant' Agostino lo invoca:

„Secretissime et praesentissime!” (Conf. I,4)

Nella Tradizione cristiana Dio ci propone il santo „gioco a nascondino” che Dio ci propone (BEHLER 1984, pp. 127-151).

\subsubsection{Perpetua e Felicitas}

La più bella testimonianza di che cosa fosse per i primi cristiani la mistica del gioco celeste si legge negli Atti delle martiri cartaginensi Perpetua e Felicità, nelle parole delle annotazioni diaristiche del martire Saturo, ricavate dall'editore degli Atti (forse Tertulliano stesso). Questi in visione contempla l'ingresso delle martiri in Paradiso le quali vengono condotte da quattro angeli al trono di Dio e Colui appare loro in aspetto d',,uomo canuto dal volto giovanile” accarezzando loro la guancia in segno di divin saluto. Accanto al trono stanno quattro uomini che dicono ai beati: Ite et ludite, ,andate $e$ giocate!". E Saturno dice a Perpetua:

„Ecco, hai quanto desideravi.”

Perpetua conclude il dialogo celeste:

„Deo gratias, ut quomodo in carne hilaris fui, hilarior sum et hic modo”, „rendo grazie a Dio. Come fui lieta un tempo nella carne, ecco, qui sarò ancor più lieta." (RAHNER 1969, p. 59)

\subsubsection{Proverbi 8,30-31 e i commentari patristici}

Nell'ottavo capitolo del libro dei Proverbi vengono descritte con particolare risalto la natura e l'azione della Sapienza. Il ruolo di quest'ultima nell'opera della creazione è descritto in modo grandioso e dettagliato:

„Quando Dio fissava i cieli, io ero là; quando tracciò un cerchio sull'abisso;

Quando stabilì al mare i suoi limiti, sicché le acque non ne oltrepassassero la spiaggia; quando dispose le fondamenta della terra."

$(\operatorname{Pr} 8,27-29)$

La Sapienza divina si presenta come un bambino il cui modo di agire, il gioco non è un gioco momentaneo ma un gioco continuo:

„Allora io ero con lui come un bimbo, ed ero nella delizia ogni giorno;

Giocando davanti a lui in ogni istante, giocando sul globo terrestre, trovando le mie delizie con i figli dell'uomo." (Pr 8,30-31)

Con tale descrizione della Sapienza che rappresenta l'azione divina nel mondo e sul mondo i saggi dell'Antico Testamento hanno preparato le vie alla rivelazione neo-testamentaria del Cristo, la 
Sapienza incarnata. La descrizione del libro dei Proverbi si adatta perfettamente al Verbo di Dio fatto carne: Egli „gioca in ogni momento” il gioco divino dell'amore e pone le sue delizie nello stare con i figli degli uomini (cfr. BEHLER 1984, p. 138).

Per cogliere tuttavia le infinite risonanze della Sapienza giocante è forse necessario risalire al termine ebraico hokma (radice $\mathrm{khkm}$ ) che i Settanta traducono ricorrendo alle parole sofia, sofos, ecc., termini che in questo contesto vanno precisati e approfonditi. Innanzitutto tale radice ebraica appare in altri due passi dell' Antico Testamento e indica sempre un gioco danzato o una danza esprimendo in tal modo la caratteristica della concezione sapienziale di Israele: strettamente legata a Dio non si attua solo una conoscenza ma anche un comportamento radicato in Dio stesso, un'attività tutta particolare che inizia nell'interiorità, un essere davanti a se stesso, „traducendo l'intatta e intangibile felicità del proprio stato, la libertà e felicità di essere liberi e l'immagine di questa gioia indicibile, il gioco" (ADRIANI 1967, p. 10).

Nel Nuovo Testamento, come è noto, tale concezione sapienziale tipica di Israele viene ripresa e applicata alla persona del Cristo: Gesù come sapienza e sapienza di Dio in Matteo 11,19, partecipante alla creazione e conservazione del mondo, Gesù bocca della sapienza che salva, sapienza scesa sulla terra, come nel prologo di Giovanni.

Ma l'eco della sapienza ludica che gioca davanti a Dio esprimendo l'infantile gioco della creazione del mondo, sì che questo, per tale origine, manifesta il carattere di un affresco grandioso ma lieve, permane ancora per poco e come nota giustamente $\mathrm{H}$. Rahner, tale ludicità va via via persa $\mathrm{o}$ almeno non si offre in tutta la sua pregnanza e densità.

Già nella versione di Settanta, il blando gioire - un gioire che solo in senso traslato esprime la ludicità presente nell'azione dell'artista che crea- sostituì il giocare. Da allora, specie nei Padri latini della Chiesa primitiva il pensiero di una sapienza che gioca dinanzi a Dio è quasi scomparso e Rahner coglie questa trasformazione progressiva in Origene che interpreta così il passo dei Proverbi:

„Questa è la sapienza che diede sempre gioia a Dio, quando l'orbe fu compiuto: dovrebbe essere chiaro che Dio è sempre colmo di gioia."

(RAHNER 1969, p. 26)

Diversa la lettura di quel passo da parte dei Padri greci dove il valore del gioco sembra serbare intatto il suo significato e il suo senso se si ricorda il verso di Gregorio di Nazianzo:

„Poiché il sublime Logos [...] gioca. Con le immagini più variopinte egli adorna a suo piacere e in ogni forma il cosmo."

Verso che rivive poi nel commento di Massimo il Confessore che su di esso fonda ,una mistica del gioco divino" in cui si ricomprende non solo la creazione ma anche l'incarnazione (cfr. ibid., p. 28).

\subsubsection{Zaccaria 8,5 e i commentari patristici}

C'è un altro testo nell' Antico Testamento che indusse i Padri a parlare della chiesa che gioca. Il profeta Zaccaria $(8,5)$ narra una visione della futura redenzione messianica e della sua pace:

„E le strade della città di Gerusalemme saranno affollate da ragazzi e ragazze che giocano."

Questa pace di una infantilità redenta comincia a realizzarsi già nella chiesa. Infatti Cirillo d'Alessandria interpreta così il testo: 
„Ragazzi o addirittura minorenni, ragazzine, cioè la grande chiesa di coloro che non sono altro che bambini, che hanno appena abbracciata la fede. Con le loro danze quasi pneumatiche essi rendono tanto bella questa città, intendo dire la chiesa. Ad essa possiamo riferire la parola della scrittura: „Venite, giubiliamo dinanzi a Dio nostra salvezza!"”

(Commentarius in Zachariam 40 [PG 72,113 B]; RAHNER 1969, p. 50)

Fede e gioia di redenzione del cristiano battezzato, dell'eterno bambino generato da Dio, sono dunque un autentico gioco infantile e la chiesa acquista bellezza dai passi di danza il cui ritmo è segnato dal sacro Pneuma. Anche Gerolamo spiega così il testo profetico di Zaccaria:

„Le strade saranno affollate da ragazzi e ragazze che giocano. Ciò avviene solo quando le città godono di sicurezza e profonda pace, poiché allora anche la gioventù libera da doveri festeggia la gioia dello stato con il gioco e la danza. Ma è lecito applicare tutto ciò alla chiesa, di cui sta scritto: 'Si dicono cose gloriose di te, o città di Dio' (Sal 86,3). In essa la gioia dello spirito trova espressione nell'atteggiamento del corpo, e $i$ suoi figli nel triplice passo di danza diranno con Davide: 'Voglio danzare e giocare davanti al volto del Signore'."

(In Zachariam 2,8 [PL 25,1465 B]; RAHNER 1969, p. 51)

Nella sua profondità poetica e teologica questa visione della chiesa che gioca e che simboleggia l'unione di tutti coloro che sono diventati felici in Cristo si affaccia ancora una volta allo spirito di un uomo altamente sensibile alla potenza di queste immagini: Notker, il monaco balbuziente di S. Gallo, che in una delle deliziose sequenze della vigilia pasquale, la festa primaverile della chiesa, cantò:

„Ecco, sotto l'amabile / vite, o Cristo, / gioca in pace / al sicuro in giardino / tutta la santa chiesa." (RAHNER 1969, p. 51)

\subsubsection{San Tommaso sul gioco}

„Rientra in fretta in casa tua; ivi divertiti, gioca e fa quello che vuoi."

(Qo 32,15)

Questo versetto dell'Ecclesiastico fu assunto da S. Tommaso d'Aquino come motivo ispiratore di uno dei suoi Opuscula e con ardita allegoresi così fu interpretato:

„Il culto della sapienza ha questo privilegio, che nell'atto in cui si compie basta, per così dire, a se stesso. Nelle attività esterne, infatti, l'uomo necessita dell'aiuto di molte cose; nella contemplazione della sapienza, invece, egli è tanto più creativo quanto più è solo con se stesso. Perciò il Sapiente, nella massima succitata, invita l'uomo a ritirarsi in se stesso... 'E là gioca!'. Qui occorre osservare che la contemplazione della sapienza, con immagine appropriata, viene assimilata al gioco per due ragioni: anzitutto perché il gioco produce gioia, e la contemplazione della sapienza comporta la gioia più profonda; poi perché l'attività ludica non ha di mira altro, ma viene ricercata per se stessa. Questo appunto accade anche nelle gioie offerte dal sapere. Perciò l'eterna Sapienza stessa paragona la sua gioia al gioco: 'Allora io ero la sua gioia giorno per giorno, giocando davanti a lui in ogni tempo' (Pr 8,30)."

(Expositio super Boethium de hebdomadibus; RAHNER 1969, p. 1)

Nei capitoli della Summa Theologica dedicati all'homo ludens S. Tommaso ha esposto con maggior esattezza la sua etica tutta improntata ad un umanesimo meravigliosamente equilibrato (II-II, q. 168, 
a. 2). Egli si riconosce in ciò debitore sopratutto dell' Etica Nicomacheia di Aristotele ma nel commento allo stesso libro dello Stagirita aveva già proposto l'eutrapelia, quel superiore equilibrio dello spirito che consiste nel saper giocare, come meraviglioso ideale di umanità, ${ }^{18}$. Per entrambi i filosofi l'eutrápelos costituisce il giusto mezzo tra il bomolóchos che è il buffone che cerca l'allegria ad ogni costo e l'agroikos, il burbero ingrugnito in una serietà ostinata. Secondo Aristotele

„Il riposo ricreante e il gioco sereno appaiono necessari alla vita."

(Ethica Nicomacheia. 4, 14, 1128 b)

Nell'opinione di S.Tommaso

„Quindi colui che è assolutamente serio manca di virtù in quanto trascura completamente il gioco, che è necessario ad una vita umana quanto il riposo."(In decem libros ethicorum Aristotelis ad Nicamachum expositio I, IV, lect. 16)

Nella Summa Theologica la saggezza greca diviene il perno d'un'arte cristiana della vita (RAHNER 1969, p. 8). Qui S. Tommaso attinge allo Stagirita ma ancor più alla saggezza dei Padri e dei Monaci dei primi tempi della Chiesa. In un passo del secondo libro del De Musica (De musica 2, 14 [PL 32, 1116 A]) S. Agostino arriva a dire che il vero sapiente dolcemente allenta e tende il rigore del proprio spirito, ciò che appunto avviene, aggiunge S. Tommaso, mediante l'agire e il parlare giocoso (S. Th. II-II, q. 168, a. 2). E qui l'Aquinate ricorda la graziosa leggenda, letta nelle Collationes di Cassiano, dell' apostolo Giovanni ormai vecchio che giocava con una pernice (Collatio 24, 21 [PL 49, 1313 A]).

Secondo la leggenda l'evangelista san Giovanni giocava un giorno con i suoi discepoli e accarezzava una pernice. Ad un tratto vide venire verso di sé un noto filosofo in tenuta da caccia il quale si meravigliò che un uomo di tale fama si abbassasse a divertimenti tanto semplici e meschini.

„'E lei, gli disse, quel Giovanni così famoso la cui illustre reputazione ha fatto
nascere in me il desiderio vivissimo di conoscerla?'[...] 'Che cosa ha in
mano?', chiese Giovanni: 'Un arco'. 'E perché non lo porta teso?'. 'Non si
deve, se rimanesse sempre flesso, la sua forza di tensione si indebolirebbe fino
a perdersi, per cui, qualora si volesse lanciare una freccia particolarmente
potente, mancherebbe all'arco il vigore necessario.' 'Nemmeno lei si meravigli
se mi concedo questa pausa di ricreazione innocente. Se di tanto in tanto non
facessi riposare la mente dalla tensione rilassandola un po', la continuità
stessa dello sforzo la infiacchirebbe, ed essa non potrebbe più rispondere
all'urgere dell'energia spirituale.',' (BEHLER 1984, pp. 129-130)

\subsubsection{La dottrina del divin gioco della grazia elettiva}

Hugo Rahner nel suo libro ricorrendo ai testi patristici e alla letteratura mistica del medioevo dimostra che anche il passaggio di Dio in mezzo agli uomini è un vero gioco, un gioco sublime: la creazione, l'incarnazione, la redenzione, sono atti del gioco divino, della theîa paideía che deve riempirli di meraviglia e indurli alla lode. La teologia ludica invece non s'inquadra esattamente entro la dogmatica di scuola che invece era studiata tanto più vivacemente nelle segrete celle dei mistici (cfr. RAHNER 1969, p. 53).

Il mistico cristiano (solo di lui intendiamo parlare qui) è un uomo che spinge lo sguardo al di là delle cose, verso l'ineffabile. Quando leva lo sguardo alla croce del suo Signore, oltre le torturanti follie del visibile gli si aprono le luminose lontananze della sicura pace nella grazia e nella redenzione di Dio; s'inizia allora tra la sua anima e il cuore di Dio quel commercium admirabile che egli sente di

\footnotetext{
${ }^{18}$ In decem libros ethicorum Aristotelis ad Nicamachum expositio. I, X, lect. 9 e I. IV., lect. 16 (ARISTOTELIS 1949, pp. 540 e 237). Ethica Nicomacheia. 10, 6, 1176 b; 4, 14, 1128 a; 2, 7, 1008 a.
} 
essere „il gioco della grazia”, come fu cantato da Beda Venerabilis in una delle sue poesie dedicate alla croce:

$$
\begin{aligned}
& \text { „Per il credente è un gioco / essere abbracciato dalla tua gioia, / poiché tu } \\
& \text { generi molte gioie / e spalanchi le porte del cielo.” (Ibid., p. 54) }
\end{aligned}
$$

Questo spandimento di grazie è un gioco divino per il mistico perché egli lo sente come un'apertura di tutte le porte, come un abbraccio d'amore a cui viene trascinato al di là di qualsiasi calcolo. Si ripete nel fondo dell'anima ciò che un giorno accadde nell'intimo della Trinità dove ebbe inizio il gioco dell'incarnazione quando fu decisa con insondabile amore la redenzione. Matilde di Magdeburgo osò arditamente dire:

„Allora lo Spirito Santo giocò al Padre con grande dolcezza un gioco e disse: Signore, Padre caro, non intendiamo essere ancora sterili." (Ibid.)

Perciò il mistico vive la propria dedizione alla grazia come un'immersione nell'onda di una nuovissima libertà, come l'inizio di una infantile indipendenza, come l'abbandono ad un amore sconfinato. E Matilde chiede all'umanità del Signore di „,aprire l'onda che gioca, che si libra nella Trinità e di cui sola l'anima vive" (ibid.). E Dio le dice:

„La mia beatitudine l'anima la sentirà purché semplicemente silenziosa si voglia gettare nelle mie divine braccia, sicché io possa giocare con lei."

E alla monaca Altheit di Trochau Cristo dice:

„Potrei fare di te una persona importante, potresti anche essere la più potente priora che sia mai esistita in questo monastero, e anche la più beata. Ma poi non potrò più giocare con te il gioco della mia grazia come altrimenti farei. Scegli dunque quel che vuoi."

E Altheit disse:

„Signore mio, non posso fare a meno della tua grazia.”(Ibid.)

$\mathrm{Al}$ mistico è lecito parlare così del gioco della grazia perché egli si accosta al più profondo mistero teologico della grazia elettiva sapendo per esperienza che Dio dona sovranamente, in modo imponderabile e tuttavia infinitamente significante il suo amore con un atto preordinato ab aeterno, senza far violenza alla libertà spirituale della sua creatura; comprende quindi che non ha da far altro che gettarsi in questa voragine pur senza perdersi e che gli è concesso essere infantile e folle. Infatti il canone fondamentale di questo divin gioco della grazia suona: „Chi perde vince”, come scriveva il converso spagnolo mistico Alonso Rodriguez S.I. nel suo trattato infantilmente profondo dal titolo Giochi di Dio con l'anima (ibid.). Ciò che assume figura in primo piano - sia esso destino, sofferenza o partecipazione cristianamente intesa all'annullamento in apparenza insensato sulla croce - è per il mistico che scruta al di là di tutti i veli il gioco miracoloso pensato dall'amore eterno con quella inventività e quella cura che solo l'amore conosce.

\section{„Il gioco di Dio varia col variar degli uomini”}

- dice il mistico Venturino da Bergamo (ibid.). Non è dunque il pensiero trascurabile di una monaca qualsiasi quello di Teresa di Lisieux che si augura di essere un semplice giocattolo,

„una palla senza valore che i bambini scagliano a terra oppure abbandonano in un angolo, ma che potrebbe anche essere stretta al cuore, se facesse piacere" (ibid., p. 56).

Anzi, qui è sottintesa una visione fondamentale e semplicemente evangelica dell'essenza della grazia: su questo piano sublime, ormai nella zona della santità, si rinnova ancora una volta nell'uomo quell'unità tra serietà e gioco di cui già Platone aveva avuto un'intuizione: far tutto e pur stimarsi meno che niente secondo la parola del Signore: 


\title{
„E quando avrete fatto tutto quanto era prescritto dite: 'Siamo servi inutili'.”
}

(Lc 17,10)

Un colto pensatore cinese ha sentito profondamente questo misticismo del gioco della grazia leggendo Teresa di Liseux e ha scritto:

„Come Shakespeare e Lao-Tsu, anche Teresa è sensibile e semplice, tanto da tornare a quella ingenuità che conosce il valore della follia."

(Ibid., p. 55)

Walter Nigg ha ragione quando afferma che il desiderio di essere un giocattolo di Dio ,rivela una musica del cuore di cui solo una santa può disporre" (ibid.). Vi si cela la più profonda serietà dell'ideale umano cristiano per cui il credente amorevole sa ,giocare" poiché solo chi si è rifugiato in Dio è sereno. Ad esprimere l'ultimo mistero del cristiano spoudogeloios non si può forse trarre dagli intimi tesori di questa miracolosa antropologia dell'homo ludens una parola più bella e più atta dell'apoftegma di un monaco orientale:

\begin{abstract}
„Abbreviare il sonno notturno ed impiegar bene le ore del giorno, non risparmiare se stesso e poi riprendersi, non è che scherzo, anzi serietà."
\end{abstract}

(Ibid., p. 55)

\section{Conclusioni}

L'idea apologetica di Hugo Rahner reagì all'antropologia homo ludens presentata da Johann Huizinga, puntando sulle origini storico-tradizionali e teologiche di essa. Partendo da Deus ludens può essere spiegato il contesto ludico dell'esistenza dell'uomo e specialmente dell'uomo cristiano. È possibile elaborare una teologia ludica di questo genere, continuando l'apologetica di alto livello culturale iniziato da Hugo Rahner e allargandola non soltanto nei confronti dell'umanismo di Huizinga ma anche nei confronti delle filosofie di gioco ciniche.

\subsection{Deus ludens - homo ludens}

I tre livelli ben distinti dell'interpretazione del gioco sono allo stesso tempo in stretta connessione: si tratta del gioco interpretato come „,evento separato del mondo”, come „,comportamento interno dell'uomo" e come „gioco ontologizzato". La connessione tra il gioco ed il cristianesimo può essere riassunta in base a questi tre aspetti d'interpretazione. La definizione del gioco interpretato come „evento separato del mondo è composta da nove punti. Tale livello d'interpretazione del gioco è legata strettamente alle azioni liturgiche, interne al mondo cristiano. Dal punto di vista della fenomenologia e della liturgia cristiana si può dire veramente che essa non può essere definita come attività producente ma come una sensazione solenne, un'immensa identificazione, una tensione tra la libertà ed i legami, una demarcazione nel tempo e nello spazio. Inoltre, tra elementi essenziali del suo dinamismo interno fa parte anche la transustanziazione del mondo. Il gioco drammatico della liturgia, nel suo fisico, nella sua psicodinamica ed anche dall'ottica dell'esistenza suscita l'interessamento dell'uomo e lo coinvolge con la fede - similmente al gioco tematico - in una storia, alla storia della salvezza. Come tutti i giochi ci rendono aperti alla comunicazione con il Totale del Mondo, anche il gioco liturgico mette i protagonisti nella presenza della Realtà Arcaica (con la sua forza sacramentale lo fa realmente). La connessione del gioco liturgico con la realtà, anzi con la realtà trascendente, può essere concepita soltanto dai fedeli. Il gioco liturgico attraverso la dedizione dell'uomo derivante dalla fede e dall'amore per Dio aiuta il gioco ,naturale" della creatura homo ludens a completarsi, a transustanziarsi nel gioco divino della vita di grazia. In tal modo è possibile che il gioco liturgico, nella sua storia essenziale di salvezza, porti in sé la drammaticità completa della fede. 
Il gioco interpretato come il „comportamento interno dell'uomo” si collega prima di tutto alle attitudini ludiche scoperte nella personalità dei santi e dei mistici. Nel caso dell'uomo vivente in un rapporto molto profondo con Dio, è assolutamente vero che per lui l'importanza degli obbiettivi del mondo profano si riduce sino ad essere insignificante. L'atmosfera „priva di obbiettivi” del gioco diventa terreno fertile per quelli che ormai non si sentono motivati dagli obbiettivi profani insignificanti. Se però la vita dell'uomo la cui anima è dedicata a Dio si libera dai legami degli obbiettivi e dei valori profani, è chiaro che le caratteristiche dell'uomo che entra nella storia del Vangelo, saranno in questo mondo la felicità (santa), la pazzia e l'incomprensione. Il cristianesimo orientale è profondamente consapevole del valore evangelico di tale incomprensione, quando dona ai suoi grandi santi il titolo onorevole „Pazzo per Dio”. Nel mondo del cristianesimo occidentale l'attitudine ludica forse è la più appariscente nella figura di San Francesco d'Assisi. Con il suo esempio si potrà presentare il valore cristiano della serenità che nasce dalla ,serietà giocosa rasente le lacrime". Tutto ciò che viene illustrato con la figura di San Francesco d'Assisi, viene sottolineato a livello di riflessione da San Tommaso d'Aquino quando disserta sul peccato presente nella mancanza dell'attitudine ludica moderata e della virtù presente nel gioco. In base a questi mosaici, si delinea la convinzione del mondo cristiano - e soprattutto di quello dei mistici -, sebbene le esagerazioni e le manifestazioni profane del gioco comprendano il motivo del peccato, la manifestazione moderata dell'attitudine ludica modesta ed ingenua (la virtù della eutrapelia) è parte organica della spiritualità autenticamente cristiana.

Il gioco ontologizzato all'interno dell'universo della fede cristiana, in connessione prima di tutto alla raffigurazione divina della creazione e dell'essere umano, è concepibile attraverso la simbolica del gioco. Il livello più globale d'interpretazione del gioco, valido in questo caso, è stato descritto con una definizione generalizzata secondo la quale si tratta di una forma esistenziale solenne nella sua manifestazione, ,muovente di qua e di là" ma pure aperta, sottomessa ad un certo ordine e quanto all'obbiettivo „semovente”. Abbiamo già parlato in precedenza dell'idea di Gadamer sulla connessione stretta tra le opere d'arte e l'essenza del gioco. A questo punto possiamo applicare i risultati di essa all'opera della creazione come il capolavoro del Creatore divino. Nella teologia della creazione la caratteristica indicata come autonomia della creatura è fortemente in linea con l'apertura e la dinamicità automanifestante e semovente del gioco, nello stesso tempo però l'ordine della regolarità dei movimenti esprime chiaramente la destinazione della creatura a Dio, la rigorosità della legge nella lingua metafisica del gioco. Il Deus ludens quindi, per il suo piacere, per la sua immagine e somiglianza crea il homo ludens e come ,aggiunta" l'Universo esistente nel totale del gioco. Il gioco però si fa l'eco della volontà del Creatore nell'ordine delle leggi valido in vari campi e contemporaneamente - nel modo paradossale - approfitta anche della sua autonomia ricevuta da Dio. Il capolavoro divino sta nel fatto che è nata un'opera che è libera nell'immenso campo di gioco dell' autoformazione.

Si è delineato un quadro ludico-teologico convergente che spiega la forza euristica filosoficoteologica della simbolica del gioco ed il valore vitale sorprendente delle esperienze di gioco vissute quotidianamente dall'uomo nell'approccio all'attitudine ludica e seria della Santa Trinità Divina, retrostante la storia della salvezza e di quella della creazione. Alla fine, dalla prospettiva cristiana possiamo far capire che il carattere ludico della nostra vita liturgica è strettamente legato alle radici più profonde della spiritualità cristiana ed anche alla „struttura base” del capolavoro dell'essere creato.

\subsection{Il dilemma falso della serietà (tetra) e del cinismo}

La realtà contenente l'uomo insieme alle sue gioie e problemi dispone di un tentativo d'interpretazione molto vasto e noto secondo il quale ogni essere ottiene il senso della sua esistenza come un piccolo elemento del Grande Gioco dell'Universo. La gente pensa generalmente che questo 
Grande Gioco anche se è interessante con le sue sorprese ed imprevedibilità - anzi, all'uomo significa un avvenimento sollecitante anche lo specchio delle esperienze - ma forse siamo pure le vittime di un gioco assurdo dell'esistenza cosmica, estranea ed indifferente per l'uomo... Jacques Monod si esprime con una metafora indimenticabile la percezione di Dio negativa, caratteristica dell'uomo odierno. Siamo zingari vagabondi, spinti sui margini dell'Universo che è sordo ed indifferente verso la musica dell'uomo.

L'uomo odierno si pone un dilemma costringente: una possibilità è di prendere conseguentemente sul serio le cose, che conduce ad un senso di vita tragico, l'altra possibilità è di prendere le cose della vita nel modo cinico, con poca serietà, nella luce del quale però tutto si scioglie in commedia. L'unità paradossale della tragedia e della commedia sarebbe la sintesi già intuita anche dagli antichi greci, che però non può diventare in sé stessa il nostro possesso. Abbiamo argomentato che esiste una terza strada, la via d'uscita dal dilemma che passa attraverso il gioco. Congiungendoci con Hugo Rahner e con tanti altri teorici del gioco sono del parere che questa serietà del gioco si unisca paradossalmente e contemporaneamente nella trascendente ed immanente Presenza di Dio. Il maestro divino di gioco dell'homo ludens è proprio il Deus ludens che, entrando nel campo da gioco della storia della salvezza, nella serietà ludica dell' amore comunica con noi.

Nel contesto del nostro ragionamento si è già delineata la possibilità autenticamente cristiana dell'interpretazione dell'essere, nell'ambito del Concetto Grande del Gioco...

\section{References}

ARISTOTELES (1949): Ethicorum Aristotelis ad Nicomachum expositio. Marietti, Torino - Roma.

ADRIANI, M. (1967): Deus ludens. Studi e materiali di storia delle religioni, 38: 8-23.

Avedon, E. M. \& Sutton-Smith, B. (1971): The Study of Games. John Wiley \& Sons, New York.

BAGYINSZKI, P. Á. ( 1998): Az Isten, az ember és a játék (Dio, l’uomo e il gioco). Agapé, Szeged.

BALOGH, T. (1991): Lélek és játék (Spirito e gioco). Akadémiai Kiadó, Budapest.

BALthasar, H. U. von (1983): Theodramatik. vol. 1., Johannes Verlag, Einsiedeln; trad. italiana (1978): Teodrammatica. vol. 1. Introduzione al dramma. Jaca Book, Milano.

BeHLER, G. M. (1984): Il gioco di Dio. Ancora, Milano.

BELlinger, G. (1990): Nagy valláskalauz (Grande guida delle religioni). Akadémiai Kiadó, Budapest.

BERgER, P. (1969): A Rumor of Angels. Modern Society and the Rediscovery of the Supernatural. Doubleday, New York; trad. italiana (1969): Il brusio degli angeli. Il Mulino, Bologna.

BREZZI, F. (1992): A partire dal gioco. Per i sentieri di un pensiero ludico. Marietti, Genova.

Bruner, J. S.; A. Jolly \& K. SYlva (eds.) (1976): Play. Its Role in Development and Evolution. Basic Book, New York; trad. italiana (1981a): Il gioco. Ruolo e sviluppo del comportamento ludico negli animali e nell'uomo. vol. 1., La prospettiva evoluzionista. Armando Armando, Roma.

Bruner, J. S.; A. Jolly \& K. SYlva (eds.) (1976): Play. Its Role in Development and Evolution. Basic Book, New York; trad. italiana (1981b): Il gioco. Ruolo e sviluppo del comportamento ludico negli animali e nell'uomo. vol. 2., Il gioco in relazione agli oggetti e agli strumenti. Armando Armando, Roma.

Bruner, J. S.; A. Jolly \& K. SYlva (eds.) (1976): Play. Its Role in Development and Evolution. Basic Book, New York; trad. trad. italiana (1981c): Il gioco. Ruolo e sviluppo del comportamento ludico negli animali e nell'uomo. vol. 3., Gioco e realtà sociale. Armando Armando, Roma. 
Bruner, J. S.; A. Jolly \& K. SYlva (eds.) (1976): Play. Its Role in Development and Evolution. Basic Book, New York; trad. italiana (1995): Il gioco. Ruolo e sviluppo del comportamento ludico negli animali e nell'uomo, vol. 4., Il gioco in un mondo di simboli. Armando Armando, Roma.

CAILloIS, R. (1967): Les jeux et les hommes. Gallimard, Paris; trad. italiana (1981): I giochi e gli uomini. Bompiani, Milano.

CAVAlieri, R. V. (1983): Filosofia del gioco. Società Editrice Napoletana, Napoli.

ČERNÝ, J. (1971): A futballról komolyan. Kísérlet a játék fenomenológiájára (Seriomente sul calcio. Tentativo per una fenomenologia del gioco). Gondolat, Budapest. (ford. Bojtár E.)

Cox, H. (1969): The Feast of Fools. Harvard UP, Cambridge; trad. italiana (1971): La festa dei folli. Bompiani, Milano.

CREsCEnzo, G. DE (1983): Il gioco e il suo piacere. Etologia e filosofia. La Nuova Italia, Firenze.

CsÁNYI, V. (1985): Etológia (Etologia). Nemzeti Tankönyvkiadó, Budapest.

CSEPREGI, G. (1994): Találkozások a játékban (Gli incontri nel gioco). Távlatok (Rivista dei gesuiti ungheresi), 16: 177-188.

EIGEN, M. \& R. WiNKLER (1985): Das Spiel. Gröbner - Hofreiter, München - Zürich; trad. italiana (1986): Il gioco. Adelphi, Milano.

ELIADE, M. (1987): The Encyclopedia of Religion. vol. 11., Macmillan, New York.

FINK, E. (1960): Nietzsches Philosophie. Kohlhammer, Stuttgart; trad. italiana (1993): La filosofia di Nietzsche. Marsilio, Venezia.

FinK, E. (1957): Oase des Glücks. Gedanken zu einer ontologie des Spiels. Karl Alber, Freiburg München; trad. italiana (1969a): Oasi della gioia. Rumma, Salerno.

FINK, E. (1960): Spiel als Weltsymbol. Kohlhammer, Stuttgart; trad. italiana (1969b): Il gioco come simbolo del mondo. Lerici, Roma.

GADAMER, H.-G. (1960): Wahrheit und methode. J.C.B. Mohr, Tübingen; trad. italiana (1983): Verità e metodo. Bompiani, Milano.

GADAMER, H.-G. (1986): L'attualità del bello. Marietti, Genova.

GANOCZY, A. (1992): Suche nach Gott auf den Wegen der Natur. Theologie, Mystik, Naturwissenschaften. Ein kritischer Versuch. Patmos, Düsseldorf; trad. italiana (1997): Teologia della natura. Queriniana, Brescia.

GEHLEN, A. (1966): Der Mensch. Athenäum, Frankfurt; trad. italiana (1983): L'uomo. La sua natura e il suo posto nel mondo. Feltrinelli, Milano.

GRASTYÁN, E. (1985): A játék neurobiológiája. Akadémiai székfoglaló 1983. (La neurobiologia del gioco. Discorso d'insediamento accademico 1983.). Akadémiai Kiadó, Budapest.

GreshaKe, G. (1988): Der Preis der Liebe. Besinnung über das Leid. Herder, Freiburg - Basel Wien.

GuARDINI, R. (1919): Vom Geist der Liturgie. Herder, Freiburg; trad. italiana (1987): Lo spirito della liturgia. Morcelliana, Brescia.

HAMILL, T. (1991): What if soul yearns to laugh?, The Way, 31: 206-215.

HANKISS, E. (1997): Az emberi kaland. Egy civilizáció-elmélet vázlata (L'avventura umana. Lo schema di una teoria della civiltà). Helikon, Budapest.

HENRIOT, J. (1969): Le jeu. PUF, Paris.

Hough, A. M. (1997): God is not 'Green'. A Re-examination of Eco-theology. Cromwell, Leominster. 
HuizingA, J. (1938): Homo ludens. Amsterdam UP, Amsterdam; trad. italiana (1946): Homo ludens. Giulio Einaudi Editore, Roma.

Invitto, G. (1988): Sartre. Dal gioco dell'essere al lavoro ermeneutico. Franco Angeli, Milano.

Johnston, R. K. (1983): The Christian at Play. William B. Eerdmans, Grand Rapids (MI).

Kuschel, K. J. (1994): Lachen. Gottes und des Menschen Kunst. Herder, Freiburg; trad. inglese (1994): Laughter. A theological reflection. SCM Press, London.

LAFFEY, A. L. (1991): Humor and play. A biblical reflection. The Way, 31: 187-195.

LONSDALE, D. (1991): This sporting life. The Way, 31: 226-235.

MCLELlAND, J. C. (1991): A serious playfulness. The Way, 31: 196-205.

MiLlar, S. (1968): The Psychology of Play. Penguin, New York.

Moltmann, J. (1971): Die ersten Freigelassenen der Schöpfung. Versuche über die Freude an der Freiheit und das Wohlgefallen am Spiel. Kaiser, München; trad. italiana (1988): Sul gioco. Queriniana, Brescia.

MoltmanN, J. (1985): Gott in der Schöpfung. Kaiser, München; trad. italiana (1986, 1992²): Dio nella creazione. Queriniana, Brescia.

NIETZSCHE, F. (1883-1885): Also sprach Zarathustra, Ernst Schmeitzner, Chemnitz; trad. italiana (1968): Così parlò Zarathustra. In Opere di Friedrich Nietzsche. vol. 6., Adelphi, Milano.

PIAGET, J. (1951): Play, Dreams, and Imitation in Childhood. Routledge \& Kegan Paul, London. doi: $10.1037 / \mathrm{h} 0050489$

RAHNER, H. (1952): Der spielende Mensch. Johannes Verlag, Einsiedeln; trad. italiana (1969): L'homo ludens. Paideia, Brescia.

SCHILLER, F. (1794): Briefe über die ästhetische Erziehung des Menschen, mit erklärenden Anmerkungen. Tübingen; trad. italiana (1971): Lettere sull'educazione estetica. Armando Armando, Roma.

SCHNEIDER, T. (ed.) (1922): Handbuch der Dogmatik. Patmos, Düsseldorf; trad. italiana (1995ab): Nuovo corso di dogmatica. vols. 1-2., Queriniana, Brescia.

SMITH, P. K. (ed.) (1984): Play in Animals and Humans. Oxford UP - Blackwell, Oxford - New York.

STRAub, E. (1993): Nyugat önfeledtsége (L'abbandono dell'Occidente). Communio (Rivista Internazionale Cattolica-Budapest), 2: 22-29.

SuURmond, J. J. (1994): Het Spel van Woord en Geest. Aanzet tot een charismatische theologie. Uitgeverij Ten Have, Baarn; trad. inglese (1994): Word and Spirit at Play. SCM Press, London.

SZÉKELY, GY. (1986): A színjáték világa. Egy müvészeti ág társadalomtörténetének vázlata (Il mondo del dramma. Lo schema della storia sociale di un ramo artistico). Gondolat, Budapest.

THOMAS, AQUINAS 1984: La somma teologica. Salani, Bologna.

TueTh, M. V. (1991): To serve God wittily-humour in discernment. The Way, 31: 216-225.

TURner, V. (1982): From Ritual to Theatre. The Human Seriousness of Play. PAJ Publications, New York; trad. italiana (1986): Dal rito al teatro. Il Mulino, Bologna.

WinnicotT, D. W. (1971): Playing and Reality. Tavistock Publications, London; trad. italiana (1997): Gioco e realtà. Armando Armando, Roma.

A Magyar Nyelv Értelmezö Szótára (Grande Dizionario della Lingua Ungherese) (1986). vol. 3., Akadémiai Kiadó, Budapest. 
